\title{
Cold Cap Bubble Topology Report
}

May 2016

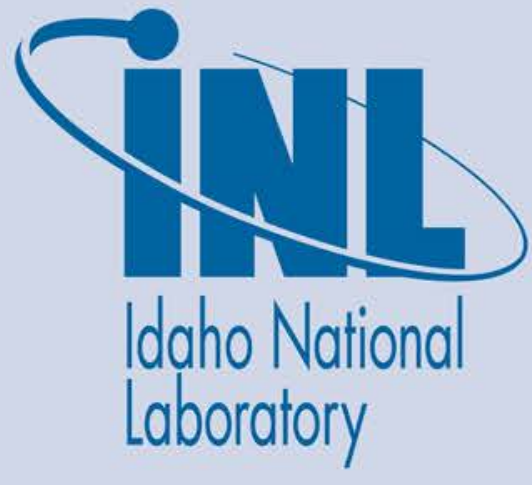

The INL is a U.S. Department of Energy National Laboratory operated by Battelle Energy Alliance 


\section{DISCLAIMER}

This information was prepared as an account of work sponsored by an agency of the U.S. Government. Neither the U.S. Government nor any agency thereof, nor any of their employees, makes any warranty, expressed or implied, or assumes any legal liability or responsibility for the accuracy, completeness, or usefulness, of any information, apparatus, product, or process disclosed, or represents that its use would not infringe privately owned rights. References herein to any specific commercial product, process, or service by trade name, trade mark, manufacturer, or otherwise, does not necessarily constitute or imply its endorsement, recommendation, or favoring by the U.S. Government or any agency thereof. The views and opinions of authors expressed herein do not necessarily state or reflect those of the U.S. Government or any agency thereof. 
INL/EXT-16-38473

Revision 0

\section{Cold Cap Bubble Topology Report}

Prepared by Lisa Mitchell, intern

Under the mentorship of Donna Post Guillen

April 2016

Idaho National Laboratory

Idaho Falls, Idaho 83415

http://www.inl.gov

Prepared for the

U.S. Department of Energy

Under DOE Idaho Operations Office

Contract DE-AC07-05ID14517 



\section{EXECUTIVE SUMMARY}

The feed composition of a high level nuclear waste (HLW) glass melter establishes the overall melting rate by influencing the thermo-physical and morphological properties of a relatively insulating cold cap layer over the molten phase. Data from X-ray tomography of laboratory-scale quenched cold caps provide insight into the distribution and morphology of bubbles, collectively known as primary foam, within this layer for various feed compositions at temperatures between $600^{\circ} \mathrm{C}$ and $1040^{\circ} \mathrm{C}$. The images were digitally segmented to capture features of interest, then processed to obtain statistics that characterize the cold cap structure, including total pellet volume, void fraction, bubble radius distribution and morphology as a function of temperature. These statistics will be used to analyze the melting behavior of the waste glass as a function of silicate content. This temperature-dependent morphological data can be used in future investigations to synthetically generate cold cap structures for use in models of heat transfer through the upper layer of a HLW glass melter, with emphasis on nuclear waste remediation application at the Hanford Site in Washington, USA. 


\section{ACKNOWLEDGEMENTS}

This work was supported by Albert Kruger of the U.S. DOE's Waste Treatment and Immobilization Plant Project of the Office of River Protection (ORP) and by the U.S. DOE Office of Science, Office of Workforce Development for Teachers and Scientists under the Science Undergraduate Laboratory Internship program. The authors are grateful to DOE's Office of Environmental Management (EM) International Program for funding of the proposal submitted from the ORP, as a part of the portfolio managed by Rodrigo Rimando of EM-HQ. We would also like to thank Michael Glazoff of the INL for fruitful discussions regarding morphological techniques for image segmentation. The work at the INL was performed by Battelle Energy Alliance, LLC under the DOE Idaho Operations Contract DE-AC07-05ID14517. X-ray tomography was performed by Professor Tetsuji Yano of the Tokyo Institute of Technology. 


\section{CONTENTS}

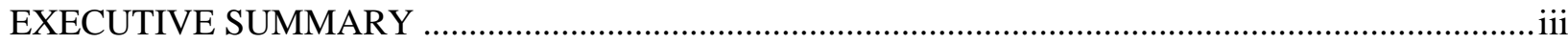

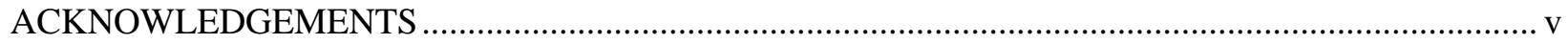

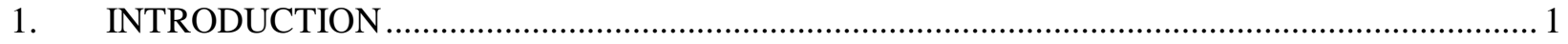

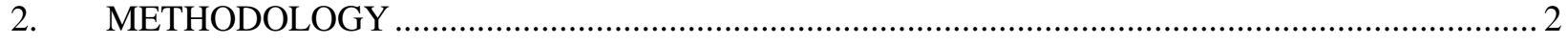

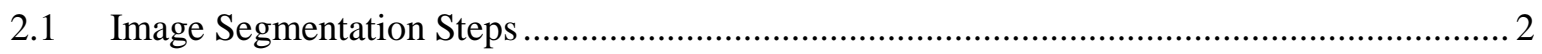

2.2 Data Extraction from Segmented Images ...................................................................... 3

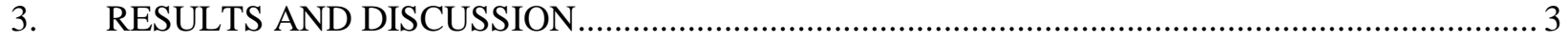

3.1 Equivalent Feature Sphere Diameter and Volume ............................................................. 3

3.2 Total Pellet Volume and Void Fraction .............................................................................. 8

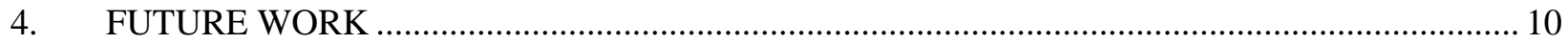

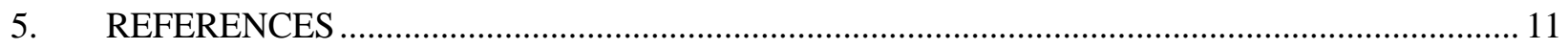

Appendix A Dream.3D Data Extraction Pipeline ................................................................................ 12

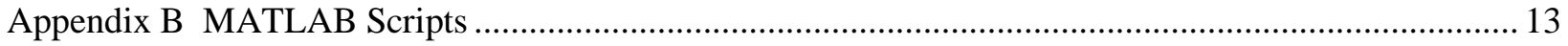

\section{FIGURES}

Figure 1. Process Flow Chart. Process Flow Chart...............................................................................

Figure 2. a) Original image A19-Original_2 at $820^{\circ} \mathrm{C}$ b) Segmentation using MATLAB

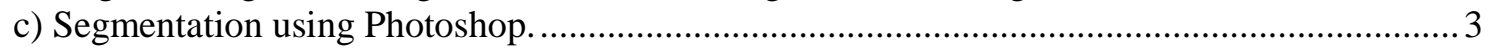

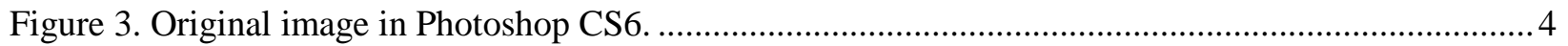

Figure 4. Center line removed using content aware fill. ................................................................. 4

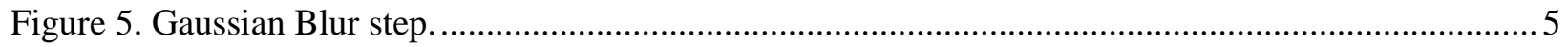

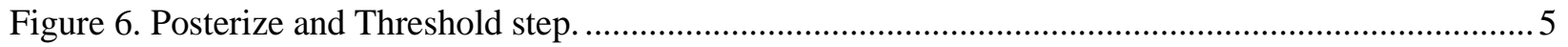

Figure 7. Correction prior to using Paint Brush Tool. ............................................................................. 6

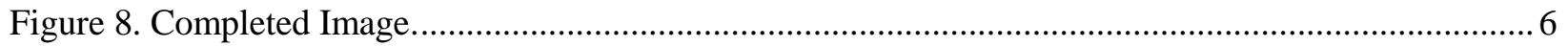

Figure 9. Bubbles identified as features by DREAM.3D...................................................................... 3

Figure 10. Equivalent Feature Diameter Boxplots................................................................................. 7

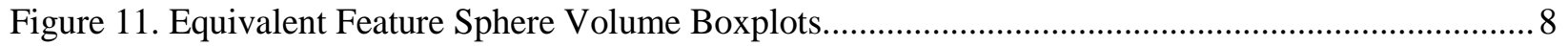

Figure 12. Total Pellet Volume Curve Fit and Extrapolation. ................................................................... 9

Figure 13. Total Pellet Volume vs. Temperature.................................................................................... 9

Figure 14. Void Fraction vs. Temperature based on 2D estimations..................................................... 10 


\section{TABLES}

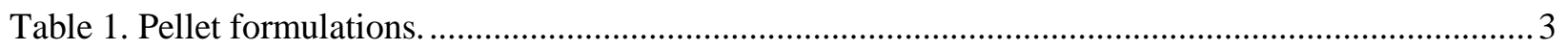

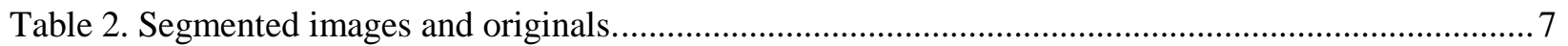

Table 3. Equivalent bubble diameter histograms with lognormal fitted curve......................................... 4

Table 4. Bubble area histograms with lognormal fitted curve................................................................. 3 


\section{Cold Cap Bubble Topology Report}

\section{INTRODUCTION}

The U.S. defense program's plutonium production operations have left the Hanford Site in southeast Washington State with over 200,000 $\mathrm{m}^{3}$ of high level radioactive waste (HLW) in underground storage tanks, many of which have been found to be leaking or structurally deficient ${ }^{1}$. The plan to secure this waste for permanent storage is through immobilization within borosilicate glass at the Hanford Waste Treatment and Immobilization Plant. The waste, in the form of an aqueous sludge containing plutonium and other radioactive nuclide-containing compounds, will be mixed with glass-forming additives such as borate and silicate frit and fed into Joule-heated ceramic melters. This mixture floats on top of molten glass where it forms a layer referred to as the cold cap in which the primary waste feed vitrification reactions occur. The off-gases from these reactions generate a primary foam layer which limits heat transfer from the molten phase below, thus reducing the rate of melting and the overall process throughput. A deeper understanding of this primary foam layer is necessary for the development of heat transfer models that more realistically represent the cold cap than has been achievable to date.

A number of investigations have focused on identifying and modeling the batch reactions that occur in the cold cap during the feed-to-glass conversion process. Hilliard and $\mathrm{Hrma}^{2}$ used optical imaging and $\mathrm{XRD}$ analysis of feed pellets heated at a rate of $5^{\circ} \mathrm{C} / \mathrm{min}$ in a quartz-glass crucible to identify silica grain size and feed composition as the primary parameters affecting primary foam production. In particular, smaller grain sizes were found to produce excessive foaming as a result of rapid dissolution of the glassformer and early onset of gas-evolving vitrification reactions. These researchers also highlighted the importance of secondary foam, in the form of oxygen bubbles produced by redox reactions in the melt, as a possible heat transfer barrier below the cold cap.

Other investigations have focused on the heat transfer and chemical modeling of the feed as it passes through the cold cap and contacts the molten glass interface, in addition to the effects of feed composition on the rate of melting. Pokorny et al. ${ }^{3}$ developed a simplified one-dimensional two-phase model of the cold cap layer that allows the vapor and condensed phases to move at different velocities through the cold cap while accounting for a number of known reactions, including those that are gas-evolving ${ }^{\mathrm{A}}$. Chun et al. used DSC-TGA measurements to analyze these off-gasses for a feed for vitrification of high-alumina HLW, relating the mass loss of the reaction mixture to the rates of production of the off-gasses in order to obtain a more complete understanding of the complex set of simultaneous reactions occurring in the melter $^{\mathrm{B}}$. These conclusions were further strengthened by the work of Rodriguez et al. that coupled the previous procedure to GC-MS measurements, thus permitting identification of the specific gases ${ }^{\mathrm{C}}$.

HLW feeds containing varying compositions of glass-forming additives have been studied for their influence on the efficiency and speed of the vitrification process by researchers at Pacific Northwest Nation Laboratory (PNNL), Savannah River National Laboratory (SRNL) and the Vitreous State Laboratory (VSL) ${ }^{4,5,6}$. A high-alumina feed composition referred to as HWI-Al-19 by VSL, or more simply as A19 by PNNL, has been shown to have considerable advantages over other feeds in the speed and efficiency of the glass conversion reactions. This feed is studied in more detail through small alterations in the mass fractions of the oxides of boron, lithium, sodium and silicon that lead to changes in the feed rheology ${ }^{7}$. The feed viscosity affects the volumetric expansion during pellet heating tests as measured with X-ray computed tomography (CT) imaging.

PNNL has also investigated porosity using X-ray CT imaging of pellets prepared using the A-0 feed. The ratio of the bulk density to the condensed density is used to calculate the porosity of the sample as a function of temperature ${ }^{2}$. This report supplements research done by PNNL on the A-19 feeds, which has focused on the external structure of the feed pellets for each of the different viscosity formulations. X-ray CT imaging allows investigation of the internal structure in a nondestructive manner, and has been used to examine quenched cold caps $^{8}$. The X-ray CT data, provided as a series of images, gives a good 
qualitative assessment of the pellet structures as a function of temperature. Morphological processing algorithms can then be used to extract quantitative data from the images, including the shapes, sizes, topology and other features ${ }^{9,10}$. Such morphological techniques have been used to study the microstructures of various solid materials ${ }^{11}$, and should be valid in analyzing semi-liquid structures, such as the cold cap.

The morphological techniques mentioned above invariably require segmentation of the raw pixel data in the image before a statistical analysis can be performed. The result of this procedure is that each pixel is assigned to a feature, which in this case would denote either a bubble or the continuous condensed phase of the cold cap. Groeber et al. outlined a framework for segmenting multi-phase material data that relies on grain orientation mismatch to identify feature boundaries, although the method is readily extendable to other criteria for inter-feature boundaries ${ }^{12}$.

The purpose of this report is to provide an overview of the analysis performed to date on the X-ray CT images of the A19 pellets obtained at the Tokyo Institute of Technology. Of the six pellet formulations, image processing has been completed on three formulations at temperatures ranging from room temperature to $1040^{\circ} \mathrm{C}$. Figure 1 illustrates the process presented in this report. INL may complete the image processing for all six sets of images, as time and resources permit.

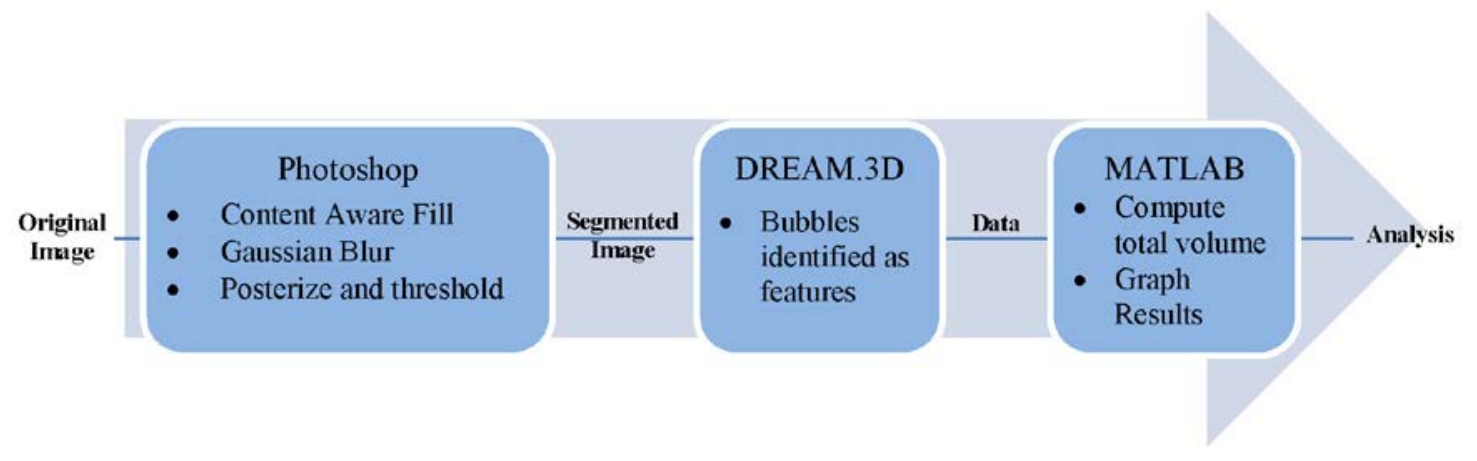

Figure 1. Process Flow Chart.

\section{METHODOLOGY \\ 2.1 Image Segmentation Steps}

Several image segmentation algorithms were explored in an attempt to gather descriptive statistics of bubble topology from the cold cap profiles. Manual segmentation supplemented with various Photoshop CS6 tools was found to yield the clearest distinction between the bubbles and background, an important requirement for subsequent analysis. Figure 2 provides an illustrative comparison of segmentation results using the MATLAB image processing toolbox and Photoshop techniques. The differences between Figures $2 b$ and 2c show that the Photoshop methods yield more highly resolved boundaries between the bubbles and the melting pellet. This section will describe the segmentation method used for pellet compositions A19-0_1, A19-1_2, and A19-original_2, the last number of each denoting the particular experiment run. Table 1 provides the chemical compositions of the pellets.

Because of the poor resolution and noise that exists in the images, it is difficult to clearly differentiate between the mass and the bubbles. Through noise reduction, thresholding, and other Photoshop CS6 techniques, this issue is mostly resolved. Additionally, the images contain a visual artifact along the center resulting from the X-ray CT measurement, rendering the image behind unusable. This portion of the image is removed from the analysis and reconstructed by approximating its neighboring pixels. 


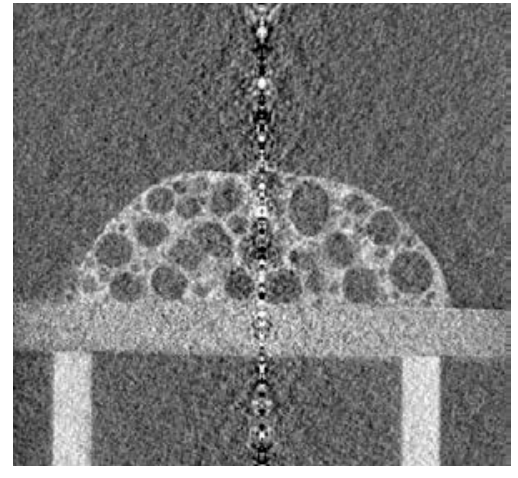

(a)

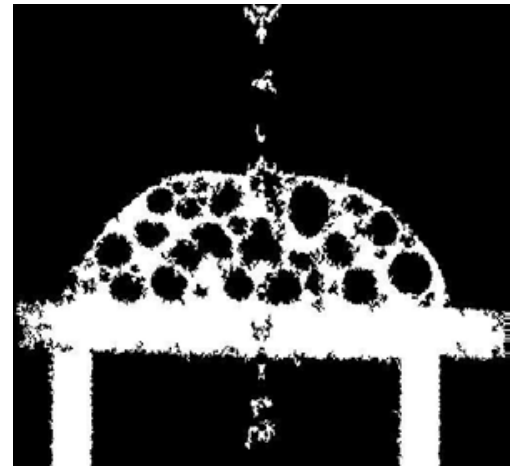

(b)

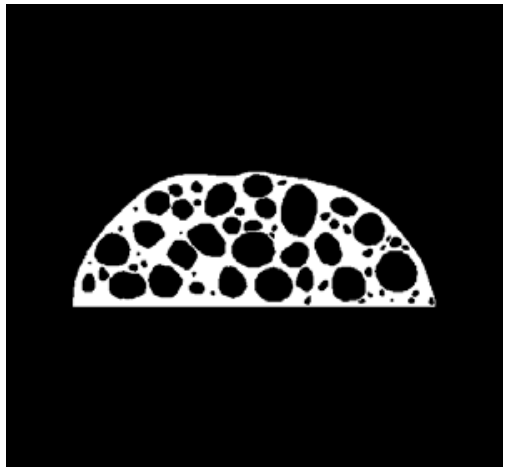

(c)

Figure 2. a) Original image A19-Original_2 at $820^{\circ} \mathrm{C}$ b) Segmentation using MATLAB c) Segmentation using Photoshop.

Table 1. Pellet formulations.

\begin{tabular}{|c|c|c|c|}
\hline Compositions (g) & A19-Original & A19-0 & A19-1 \\
\hline $\mathrm{AlO}_{3} \mathrm{H}_{3}$ & 371.79 & 371.79 & 371.79 \\
\hline $\mathrm{H}_{3} \mathrm{BO}_{3}$ & 341.59 & 431.77 & 380.87 \\
\hline $\mathrm{Bi}_{2} \mathbf{O}_{3}$ & 11.67 & 11.67 & 11.67 \\
\hline $\mathrm{CaO}$ & 10.87 & 10.87 & 10.87 \\
\hline $\mathrm{Cr}_{4} \mathrm{O}_{9} \mathrm{H}_{6}$ & 6.2 & 6.2 & 6.2 \\
\hline $\mathrm{NaF}$ & 15 & 15 & 15 \\
\hline $\mathrm{FeO}_{3} \mathrm{H}_{3}$ & 74.38 & 74.38 & 74.38 \\
\hline $\mathrm{Li}_{2} \mathrm{CO}_{3}$ & 89.22 & 112.78 & 99.49 \\
\hline $\mathrm{NiO}_{2} \mathrm{H}_{2}$ & 5.03 & 5.03 & 5.03 \\
\hline $\mathrm{FeH}_{6} \mathbf{P}_{3} \mathrm{O}_{6}$ & 12.51 & 12.51 & 12.51 \\
\hline PbO & 4.17 & 4.17 & 4.17 \\
\hline $\mathrm{SiO}_{2}$ & 221.45 & 151.45 & 190.96 \\
\hline $\mathrm{Na}_{2} \mathrm{SO}_{4}$ & 3.6 & 3.6 & 3.6 \\
\hline $\mathrm{ZrO}_{4} \mathrm{H}_{4}$ & 5.53 & 5.53 & 5.53 \\
\hline $\mathrm{CaSiO}_{3}$ & 97.07 & 97.07 & 97.07 \\
\hline $\mathrm{NaOH}$ & 19.87 & 19.87 & 19.87 \\
\hline $\mathrm{Na}_{2} \mathrm{CO}_{3}$ & 106.57 & 134.7 & 118.83 \\
\hline $\mathrm{NaNO}_{2}$ & 3.48 & 3.48 & 3.48 \\
\hline $\mathrm{NaNO}_{3}$ & 12.4 & 12.4 & 12.4 \\
\hline $\mathrm{Na}_{2} \mathrm{C}_{2} \mathrm{O}_{4}$ & 1.26 & 1.26 & 1.26 \\
\hline Sum (g) & 1413.66 & 1485.54 & 1444.97 \\
\hline
\end{tabular}

For explanatory purposes, screenshots have been provided of the segmentation process for composition A19-original_2 at $940^{\circ} \mathrm{C}$, shown in Figure 3. To improve the speed and consistency of the process, the Photoshop Actions tool was implemented, as seen in Figure 3 under 'Actions', which can record sequences of image manipulations and repeat them over a large dataset. 


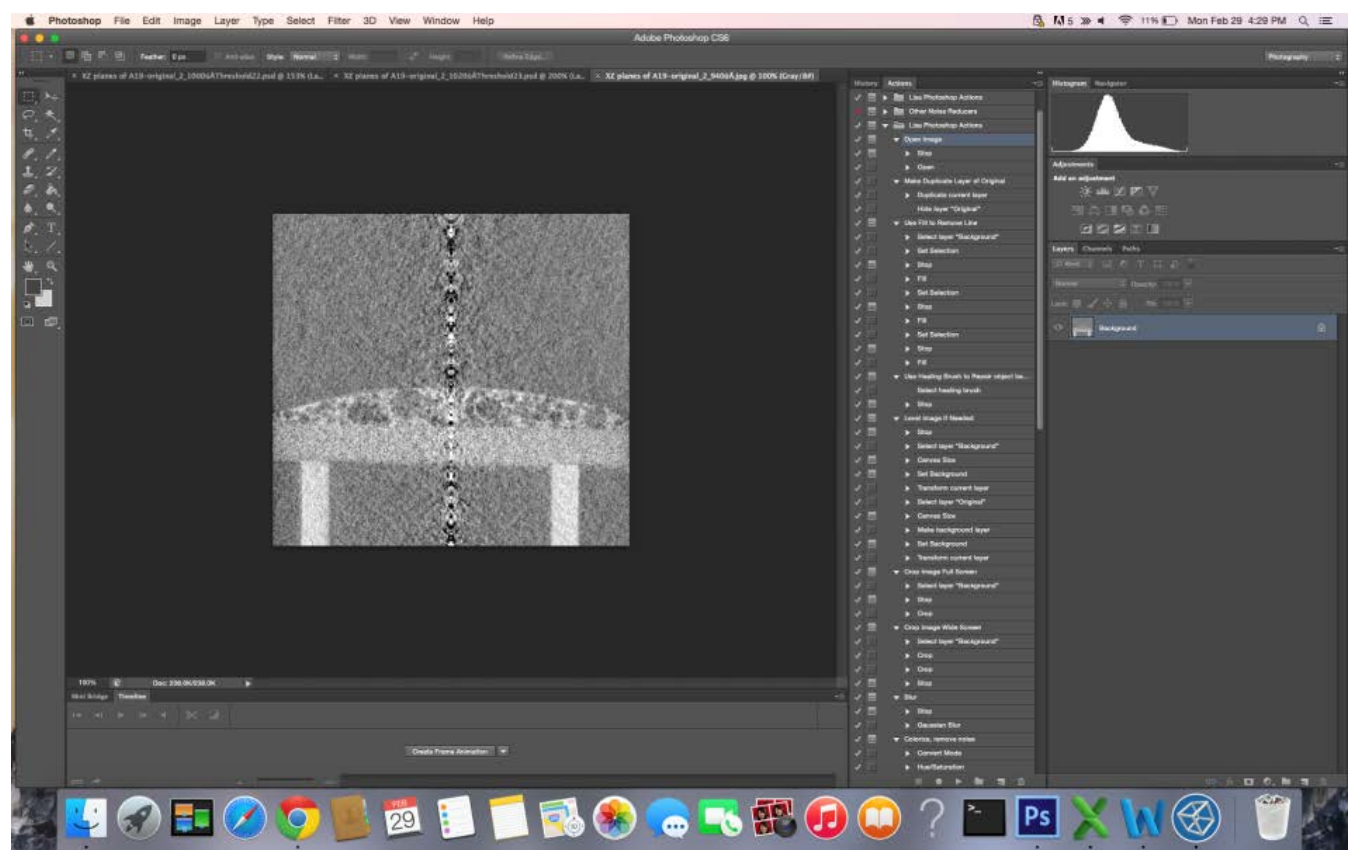

Figure 3. Original image in Photoshop CS6.

A copy of the original image is initially made in the form of a 'Photoshop layer' as seen in Figure 4 under 'Layers'. Using the Photoshop tool Content Aware Fill, the obstructing line is removed and the affected pixels approximately reconstructed by comparing to the surroundings. Gaussian Blur is performed to reduce noise in the image (Figure 5) and produce a clearer binary image in the next step, Posterization and Thresholding. In this step (Figure 6), the Posterize adjustment operation maps pixel grayscale values to the closest of three levels. Manual modifications are then made by filling color as background, bubble area, or non-bubble area, followed by a binary threshold.

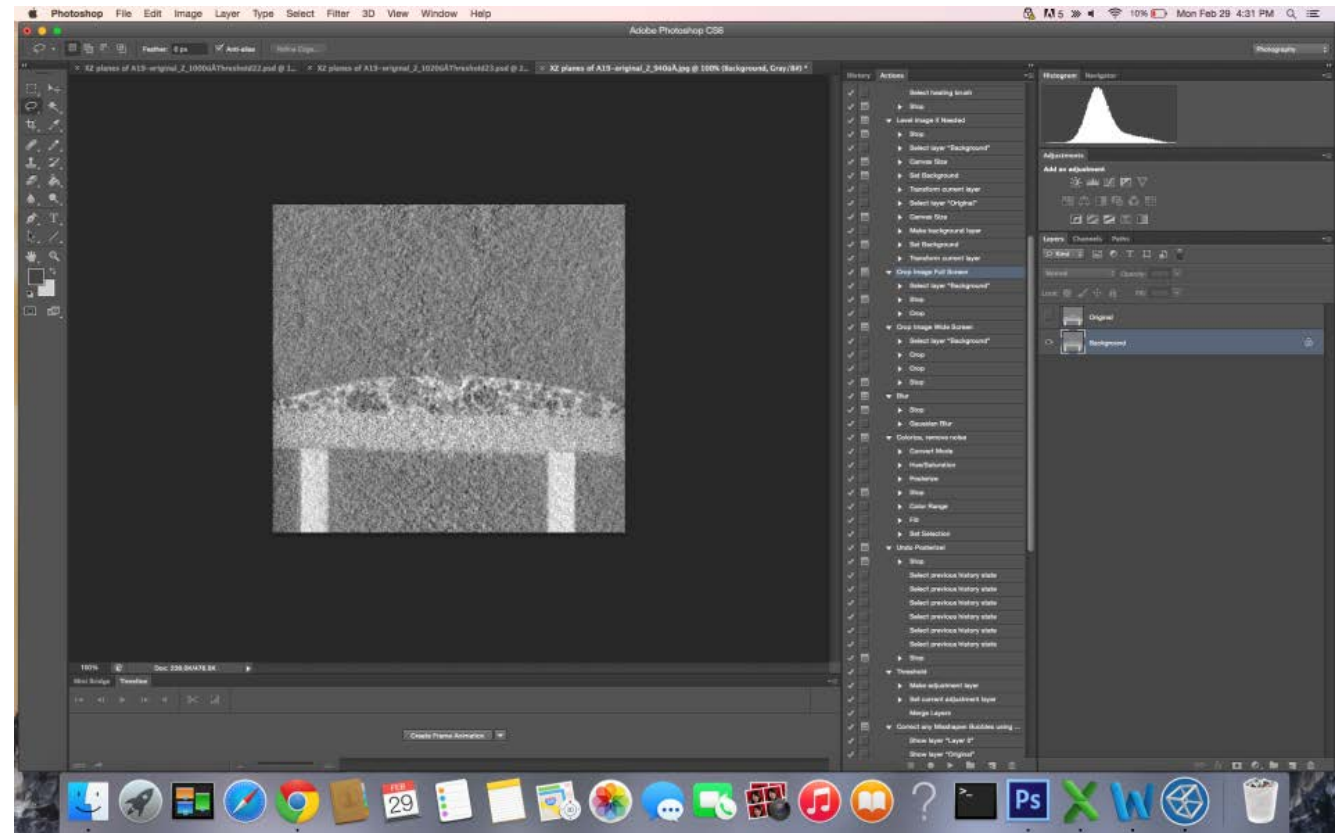

Figure 4. Center line removed using content aware fill. 


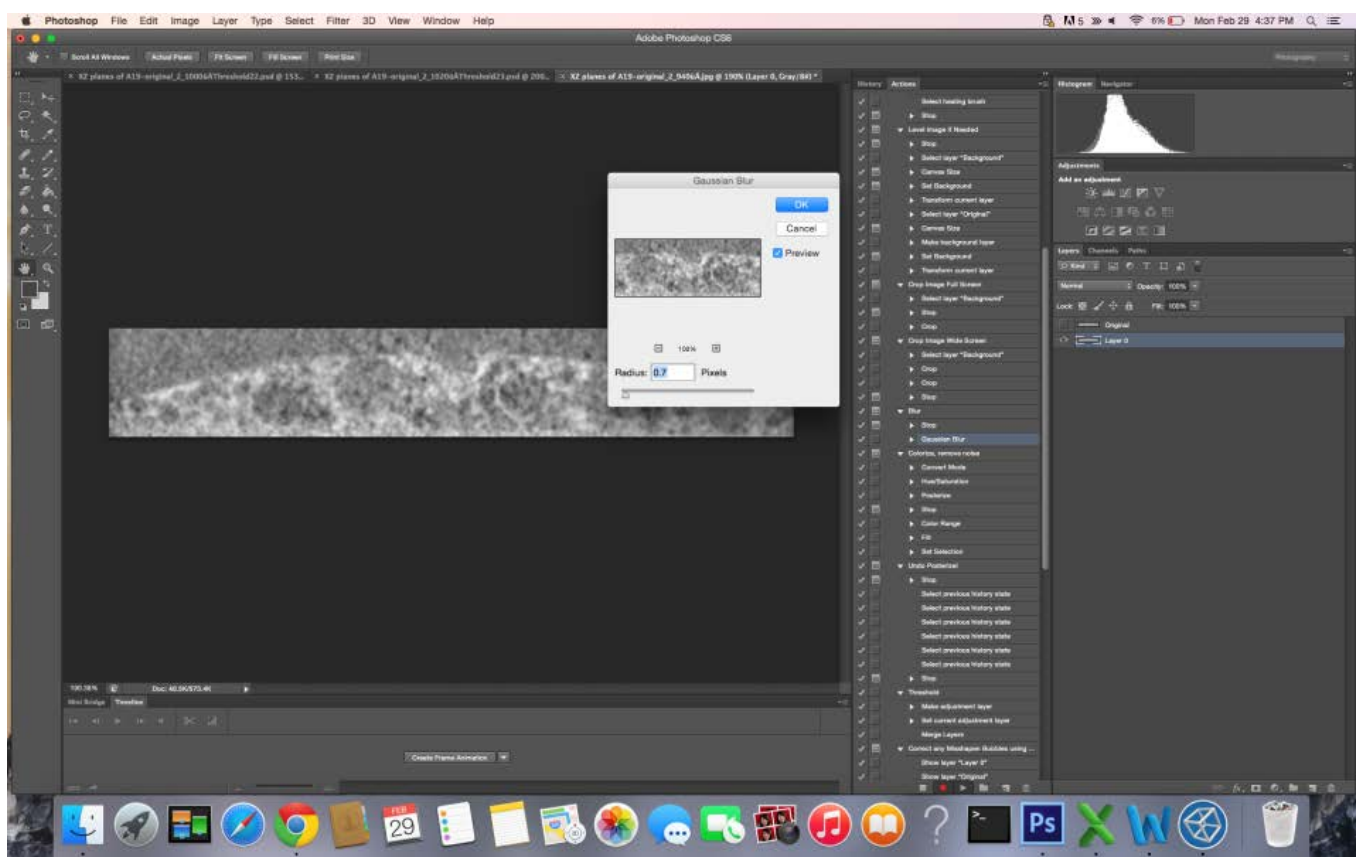

Figure 5. Gaussian Blur step.

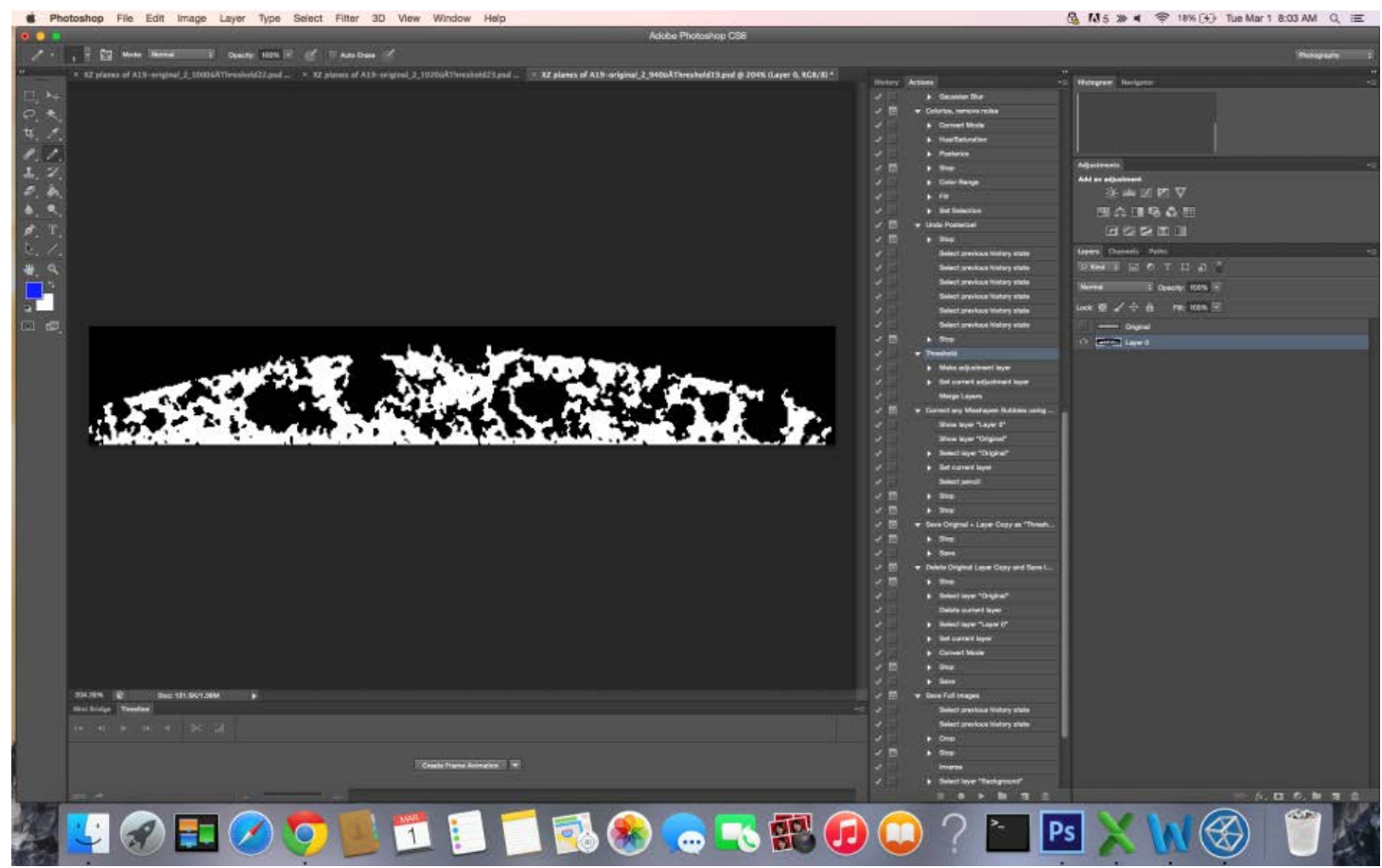

Figure 6. Posterize and Threshold step.

Finally, the Paint Brush tool is used to correct any misshapen bubbles or add any bubbles which have not been included (Figure 7). At this step, reference to the original image layer is used to retain accuracy. Lastly, Figure 8 shows the completed segmented image. Table 2 contains complete sets of both original and segmented images prepared using this method for the three pellet compositions mentioned previously. 


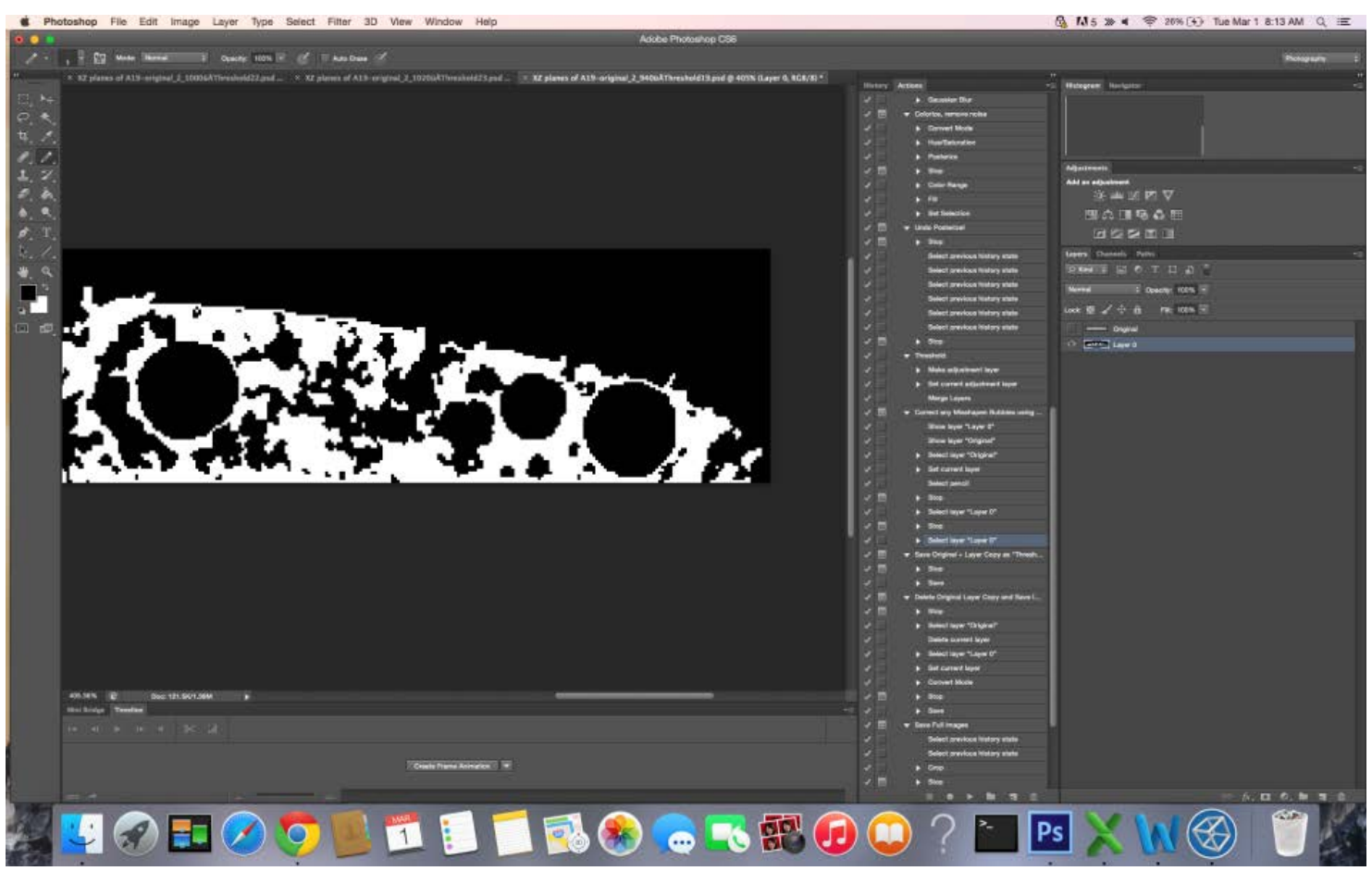

Figure 7. Correction prior to using Paint Brush Tool.

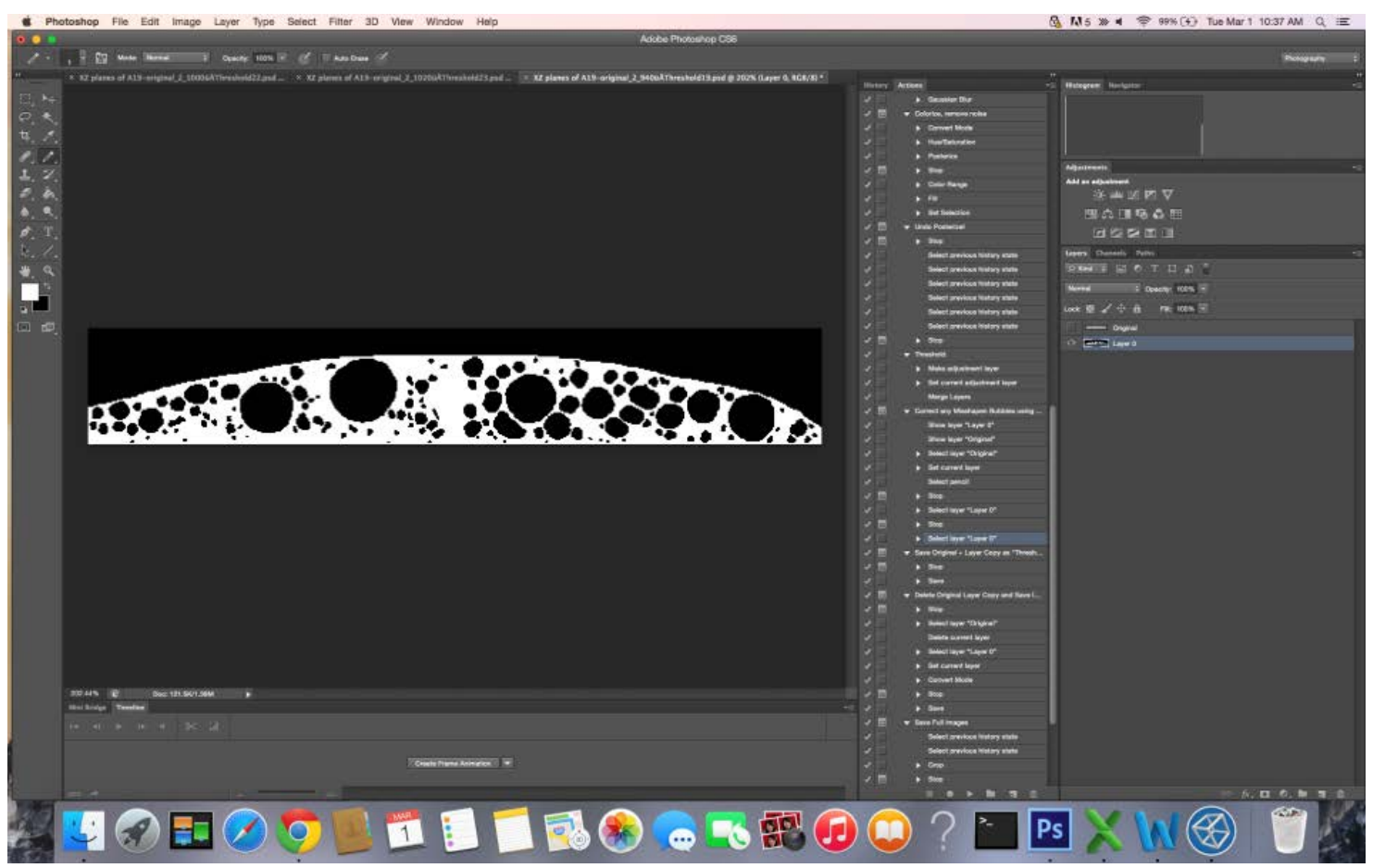

Figure 8. Completed Image. 
Table 2. Segmented images and originals.

\begin{tabular}{|c|c|c|c|}
\hline $\mathbf{T}\left({ }^{\circ} \mathrm{C}\right)$ & A19-original_2 & A19-0_1 & A19-1_2 \\
\hline Room & & & \\
\hline 600 & & & \\
\hline 620 & & & \\
\hline 640 & & & \\
\hline 660 & & & \\
\hline 680 & & & \\
\hline 700 & & & \\
\hline 720 & & & \\
\hline
\end{tabular}


Table 2. (continued).

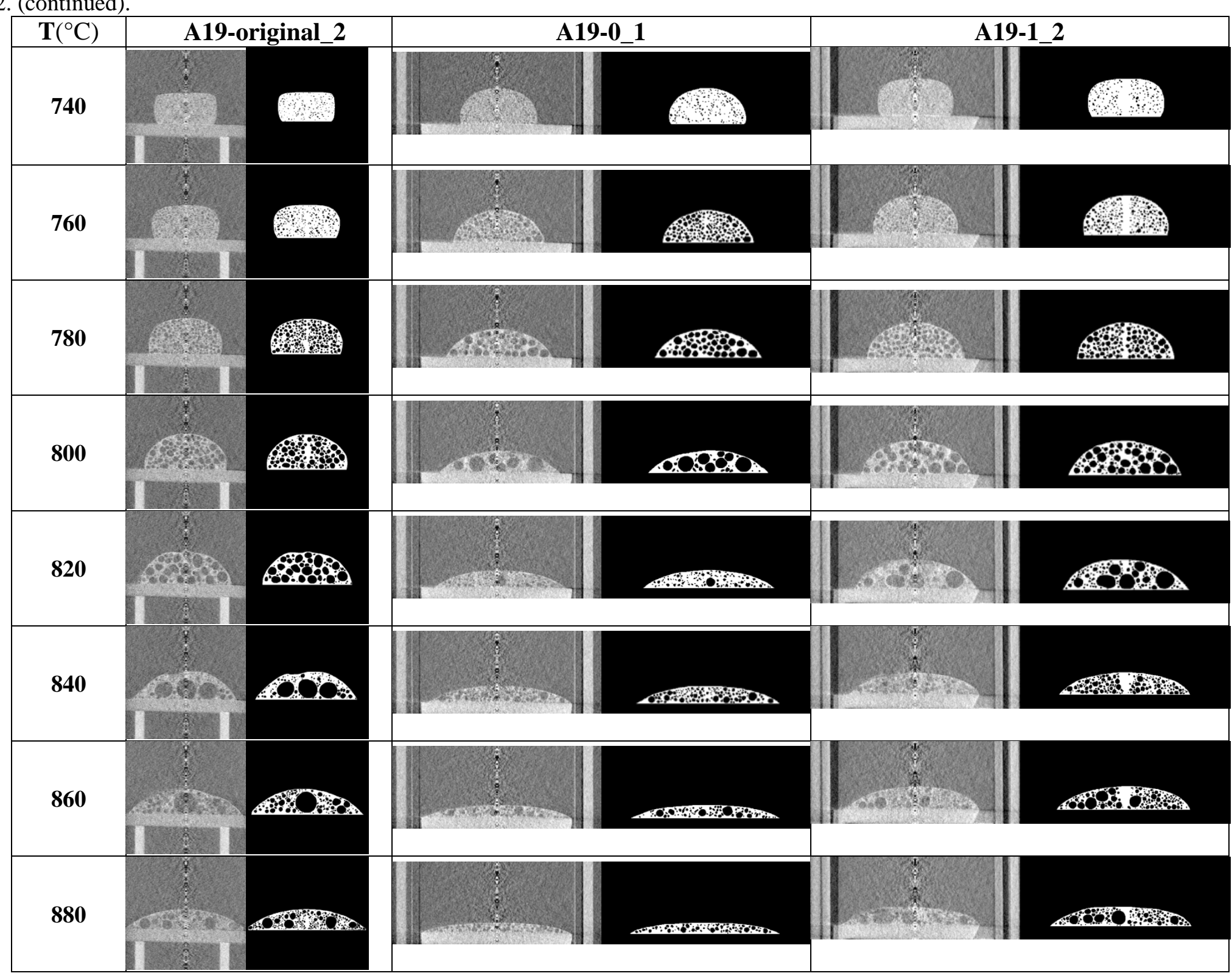


Table 2. (continued).

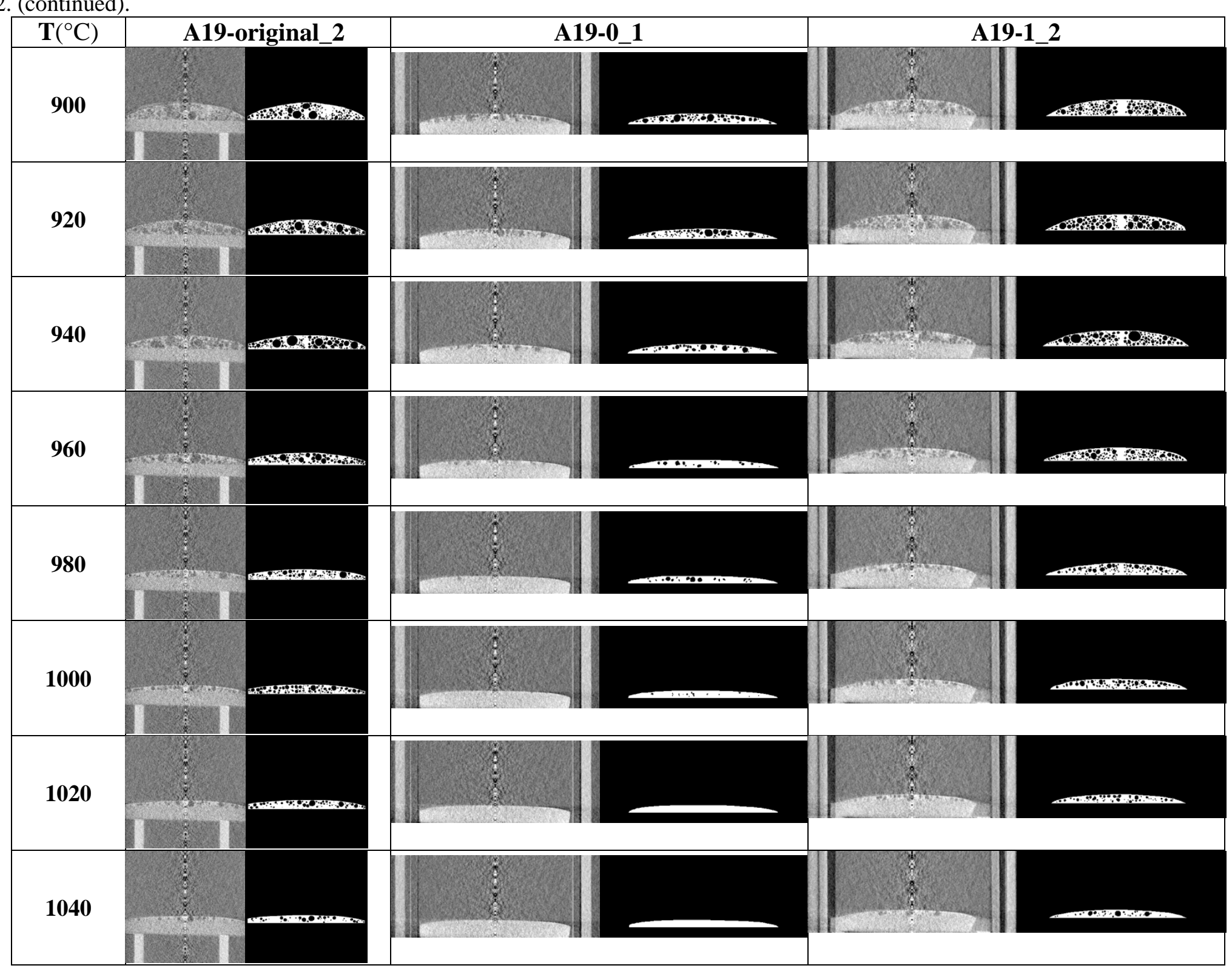




\subsection{Data Extraction from Segmented Images}

DREAM.3D is an open source software environment that allows integrated processing, characterization, manipulation, and visualization of multidimensional, multimodal data. Using DREAM.3D, features are identified from the binary images, by assigning pixels with certain grayscale values to a class, then each feature is assigned a feature identification or Feature ID. Figure 9 illustrates the Feature IDs for sample A19-original_2 at $940^{\circ} \mathrm{C}$ as viewed using ParaView (software capable of executing DREAM.3D .XDMF file visual output).

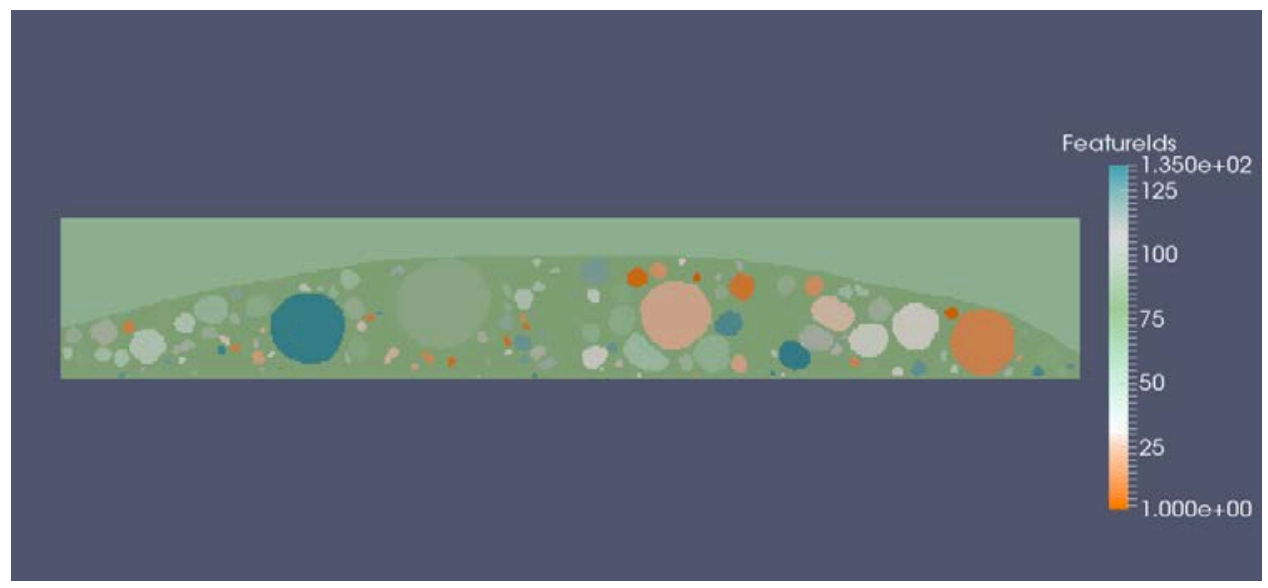

Figure 9. Bubbles identified as features by DREAM.3D.

DREAM.3D permits collection of morphological data such as equivalent feature diameter (diameter of a sphere with the same estimated volume as the feature), feature size (area of the identified feature), and the number of neighboring features. This software can also be used to generate a synthetic model of the cold cap structure for a particular pellet composition. In addition to the DREAM.3D analysis, the binary images were also run though the ImageJ software for analysis. It was found that ImageJ is unable to resolve the smaller feature IDs and missed about $10 \%$ of the total bubbles in the test cases. For this reason, additional analysis with ImageJ was not conducted.

\section{RESULTS AND DISCUSSION}

Due to the time consuming nature of the image segmentation, conclusive analysis of the data has been limited, however a preliminary analysis has been conducted. This analysis was completed in MATLAB, using the data obtained from DREAM.3D discussed previously. As DREAM.3D assigns all pixels to a class, in the identification of features both the background and solid pellet area are assigned a Feature ID. Since we are solely interested in the features identified as bubbles in this analysis, these features with the largest area are excluded. As the data obtained from DREAM.3D is in units of voxels, conversion is made to millimeters using the rates $0.079,0.079$ and $0.052 \mathrm{~mm} /$ voxel provided by Professor Yano for A19-0_1, A19-1_2, and A19-original_2 respectively.

\subsection{Equivalent Feature Sphere Diameter and Volume}

In the interest of analyzing the distribution of bubble sizes over the sequence of temperatures, histograms have been created for each image of Equivalent Feature Diameter and Feature Size (Table 3 and Table 4). These distributions have been identified to follow a lognormal distribution and have been fit with the appropriate lognormal curve using a modified version of the function histfit() (Appendix B, Subsection B-1) in order to obtain its parameters. It is important to note that no histograms are made for the temperatures from Room $-700^{\circ} \mathrm{C}$ due to no recordable bubbles present in this temperature range. This 
same effect also occurs for temperatures $1020^{\circ} \mathrm{C}$ and $1040^{\circ} \mathrm{C}$ for composition A19-0_1. Also, due to the limited number of bubbles in some of the images accuracy of the fitted curve varies due to poor sample size; for example, A19-0_1 at $1000^{\circ} \mathrm{C}$ has a sample size of 15 bubbles.

Table 3. Equivalent bubble diameter histograms with lognormal fitted curve.

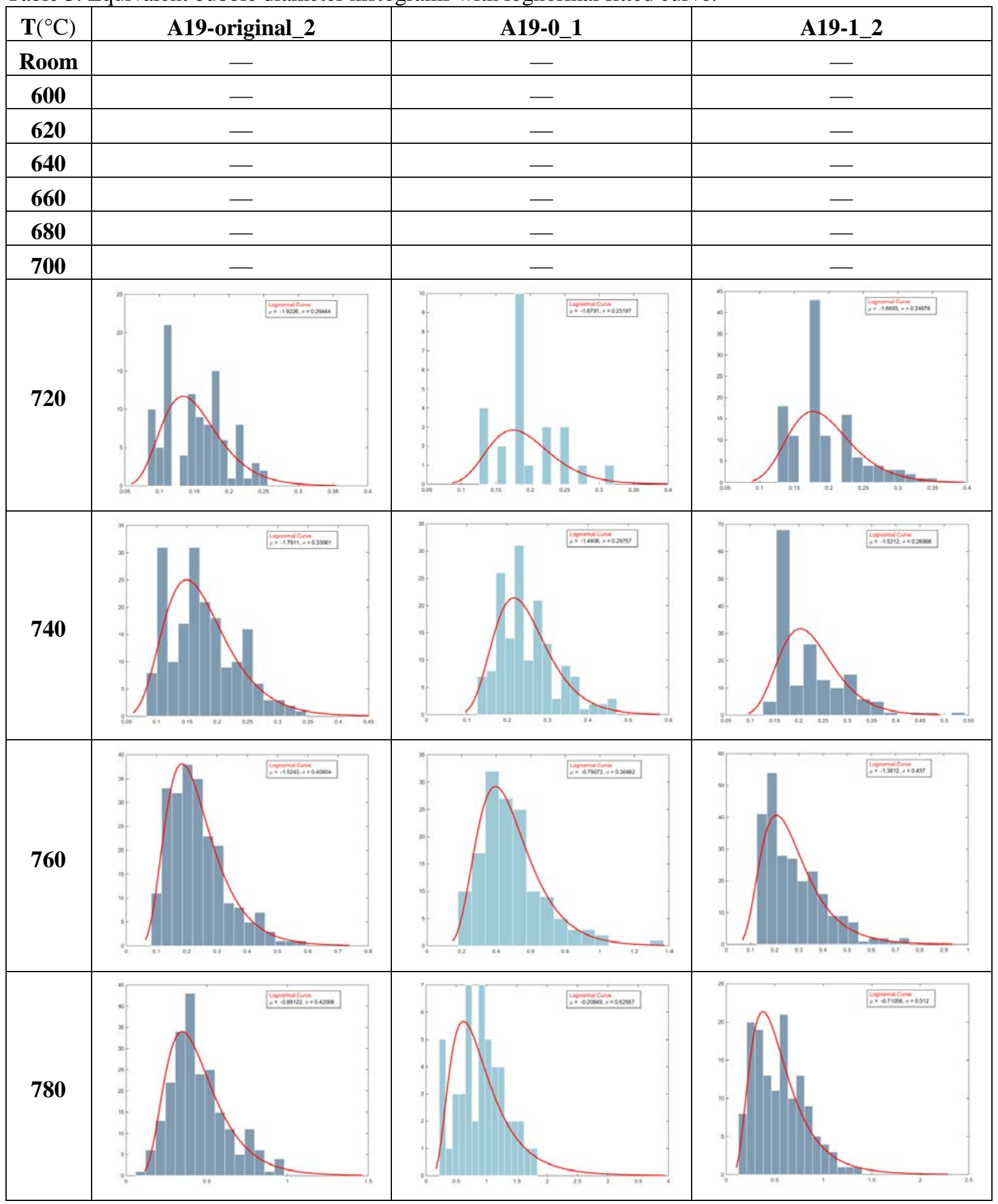


Table 3. (continued).

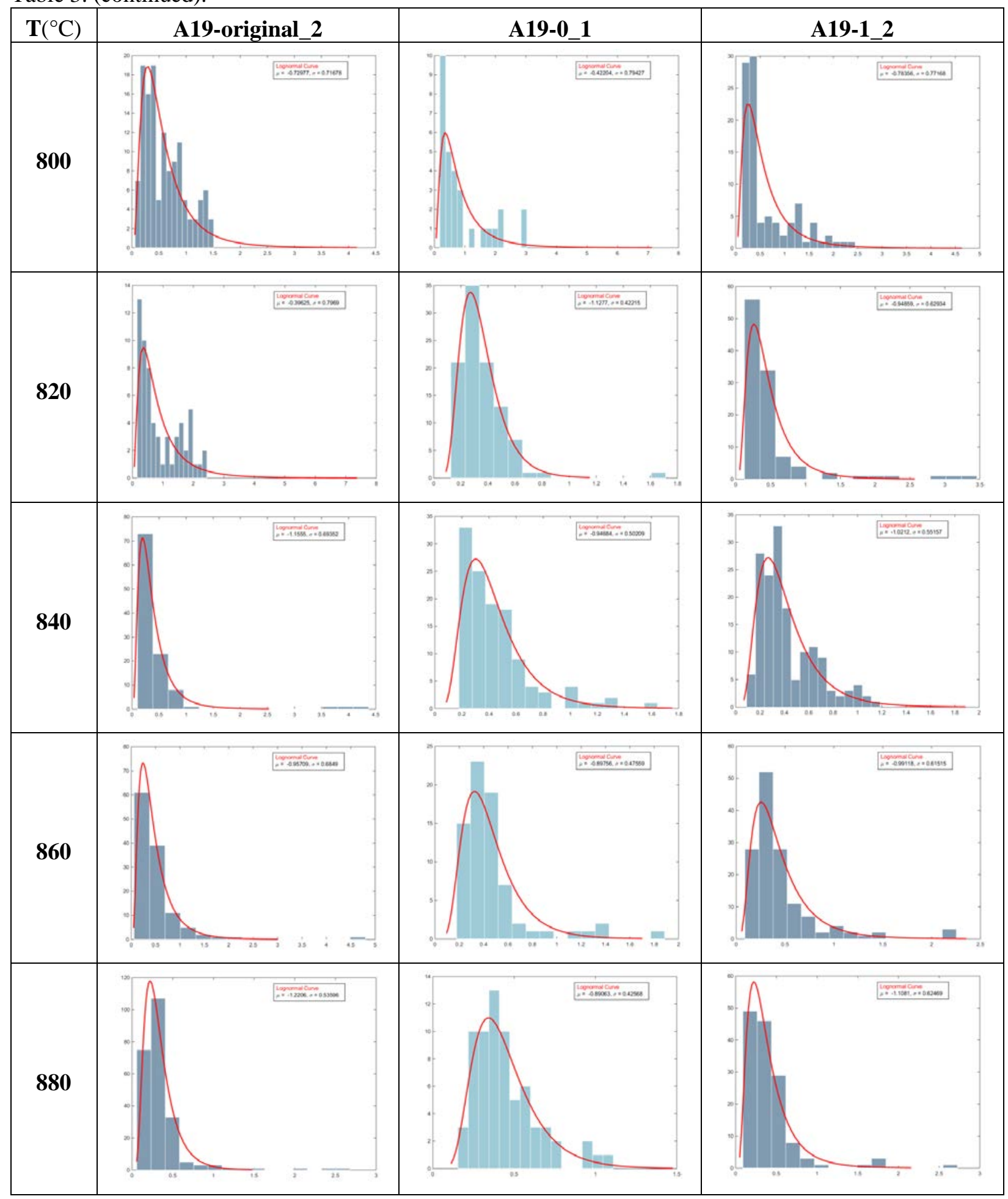


Table 3. (continued).

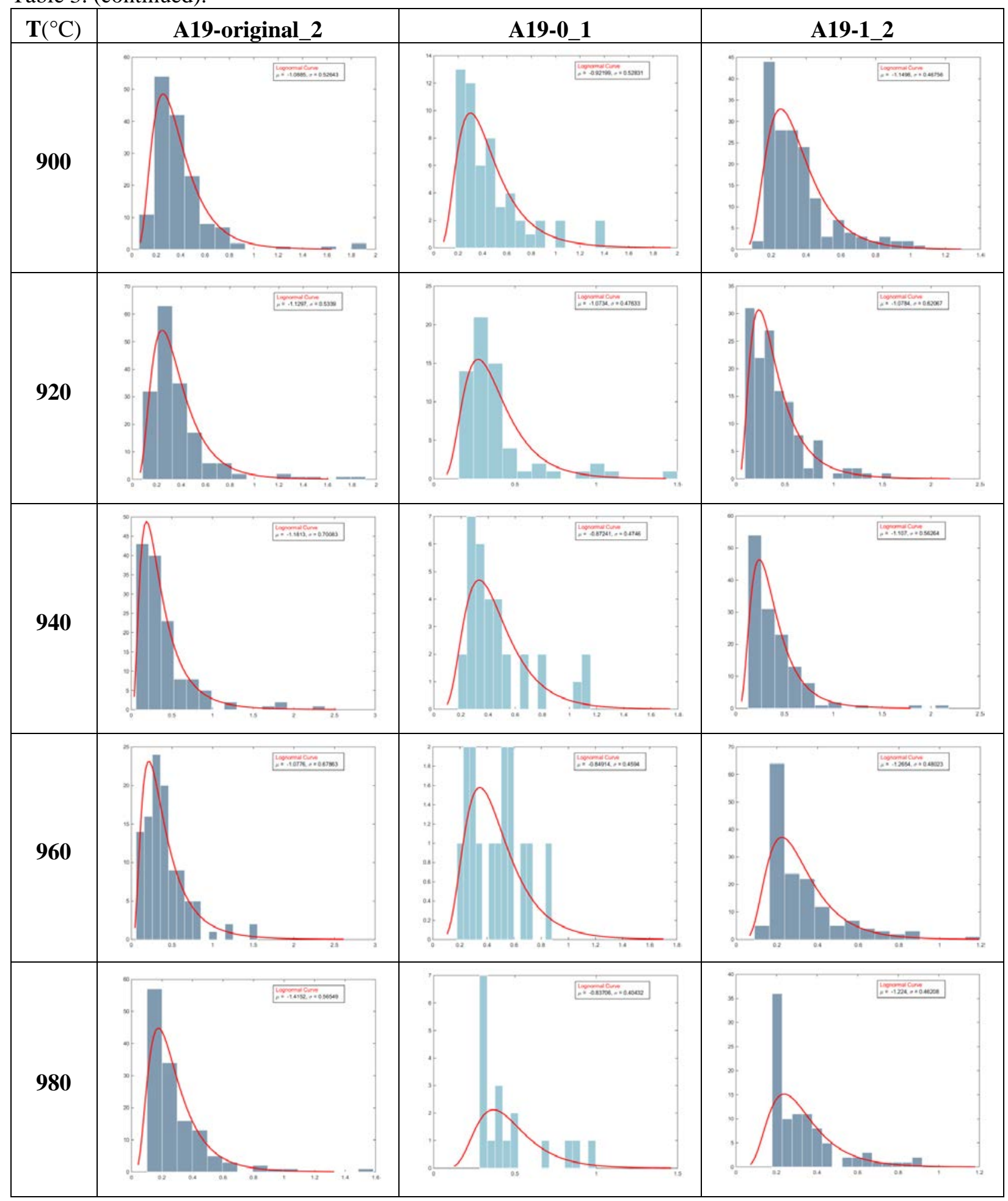


Table 3. (continued).

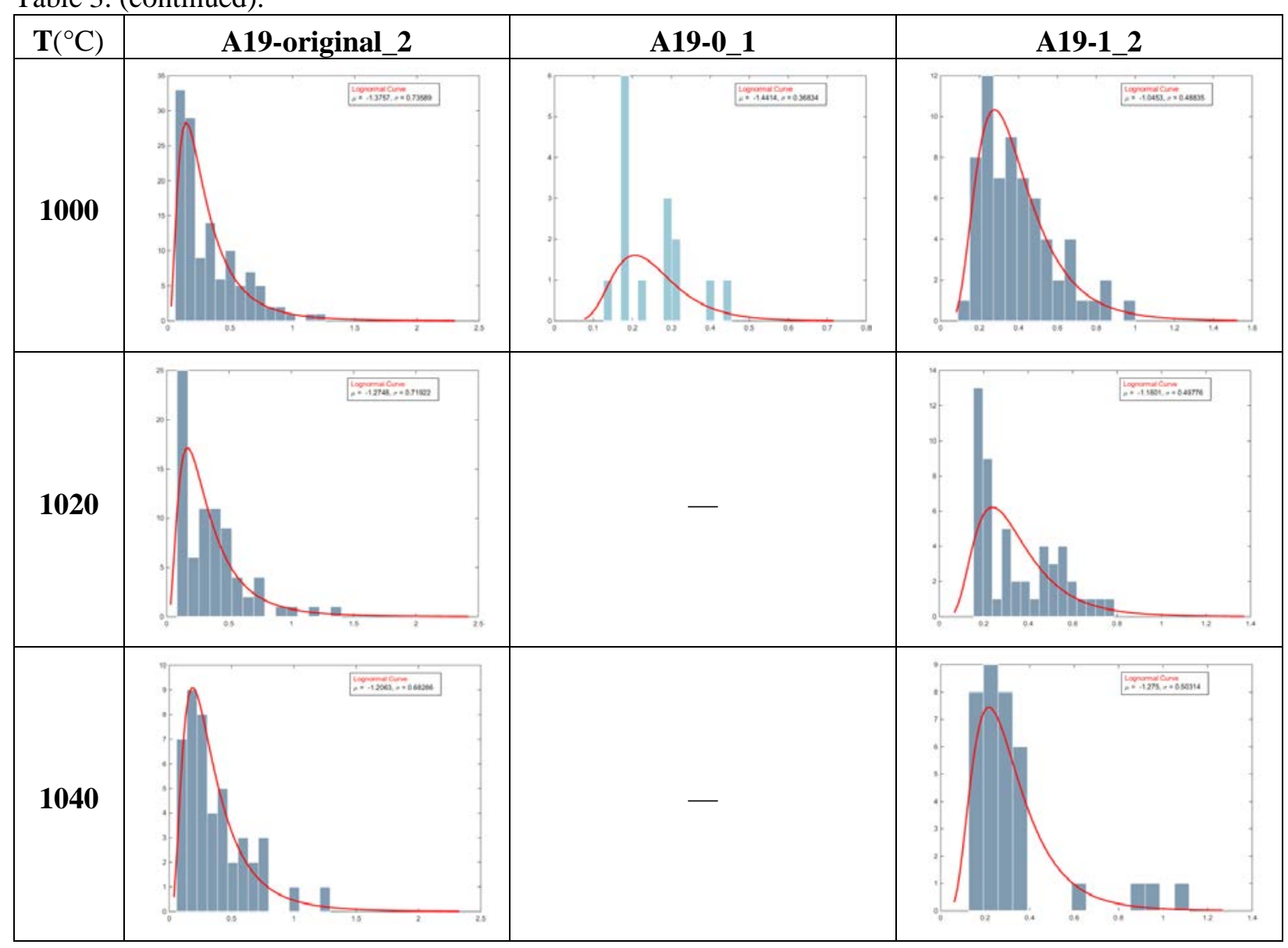

Table 4. Bubble area histograms with lognormal fitted curve.

\begin{tabular}{|c|c|c|c|}
\hline $\mathbf{T}\left({ }^{\circ} \mathrm{C}\right)$ & A19-original_2 & A19-0_1 & A19-1_2 \\
\hline Room & - & - & - \\
\hline 600 & - & 一 & - \\
\hline 620 & - & 一 & - \\
\hline 640 & - & - & - \\
\hline 660 & - & 一 & - \\
\hline 680 & - & - & - \\
\hline 700 & - & - & - \\
\hline 720 & an & 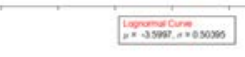 & 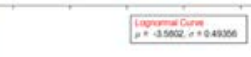 \\
\hline
\end{tabular}


Table 4. (continued).

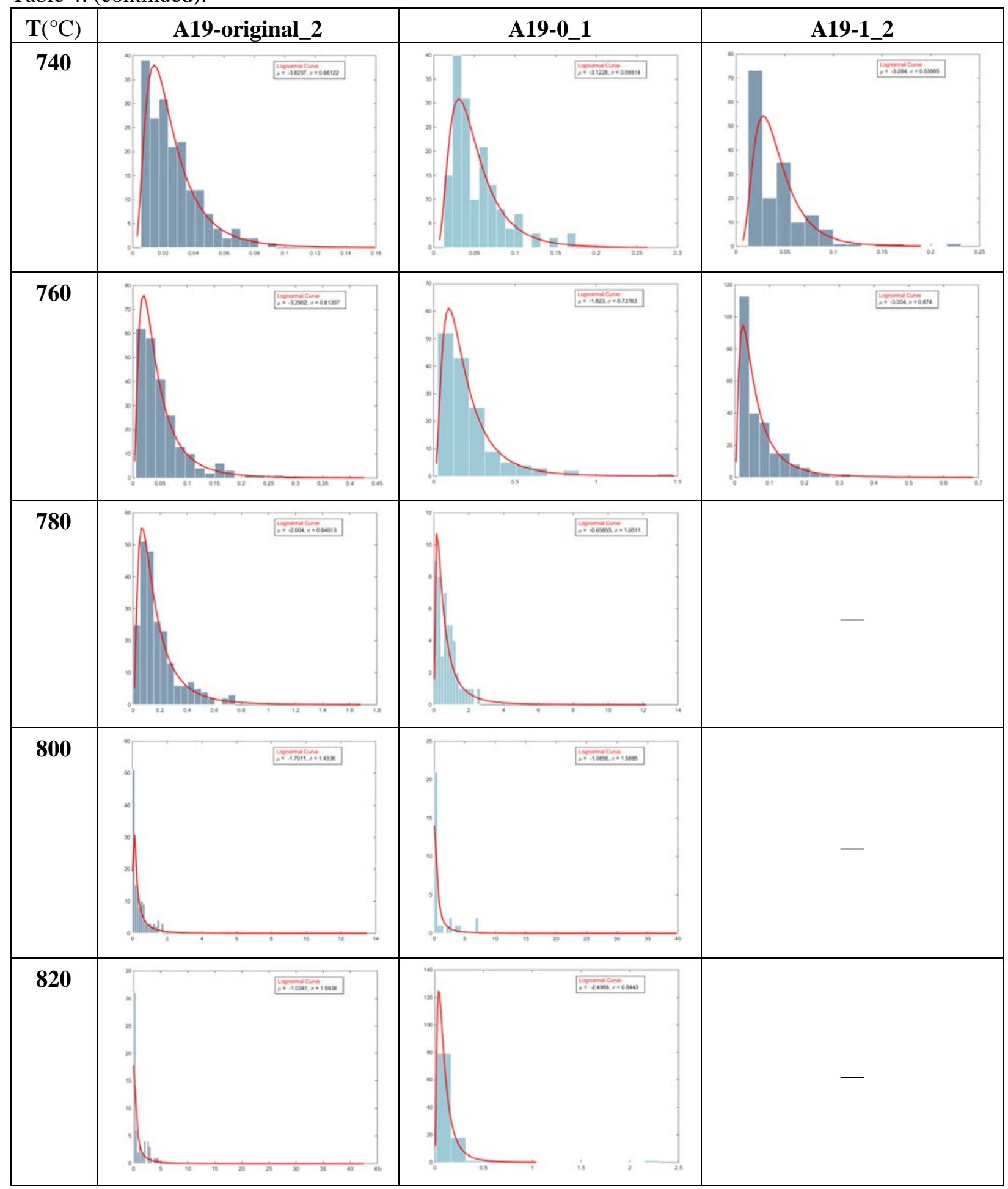


Table 4. (continued).

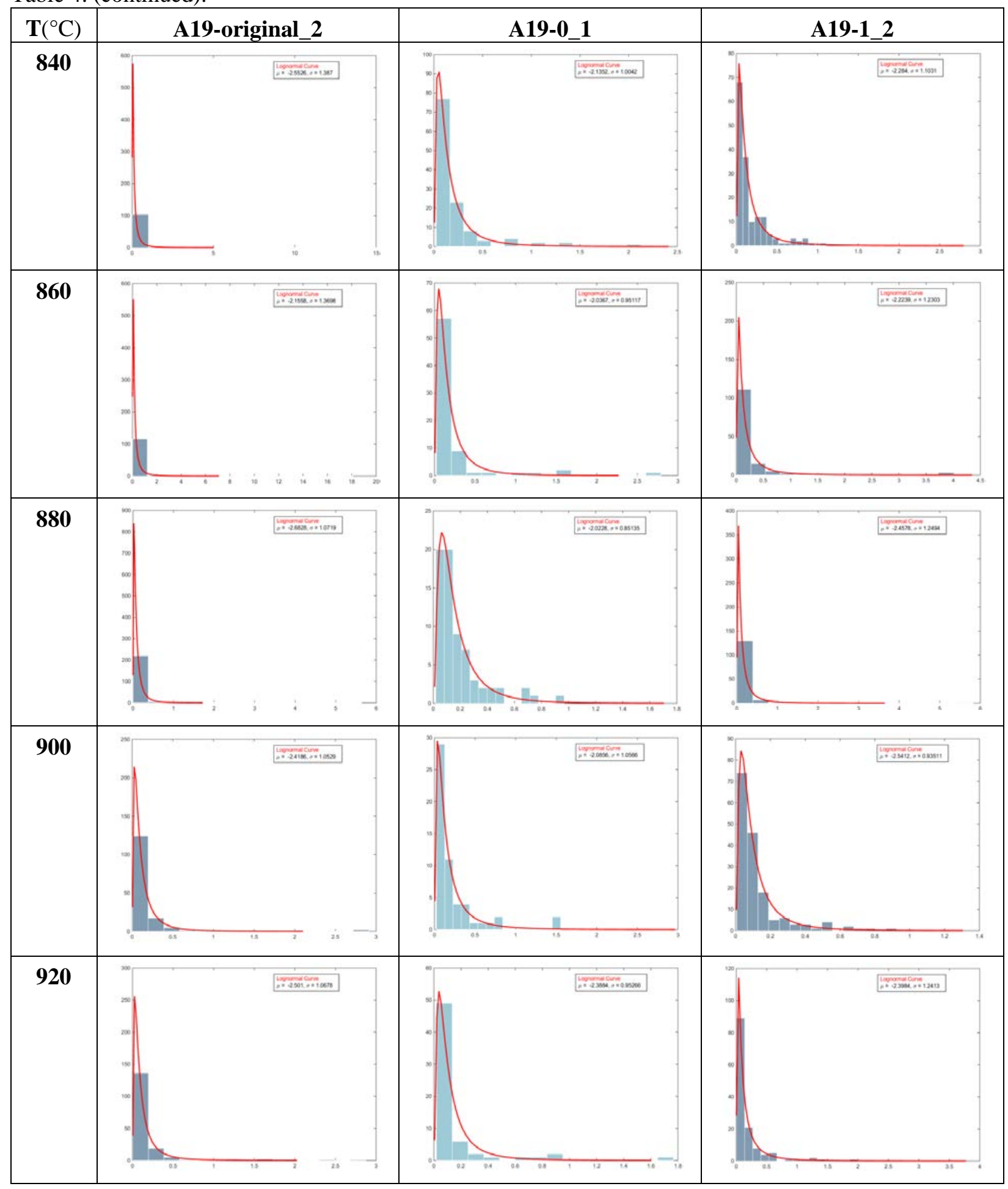


Table 4. (continued).

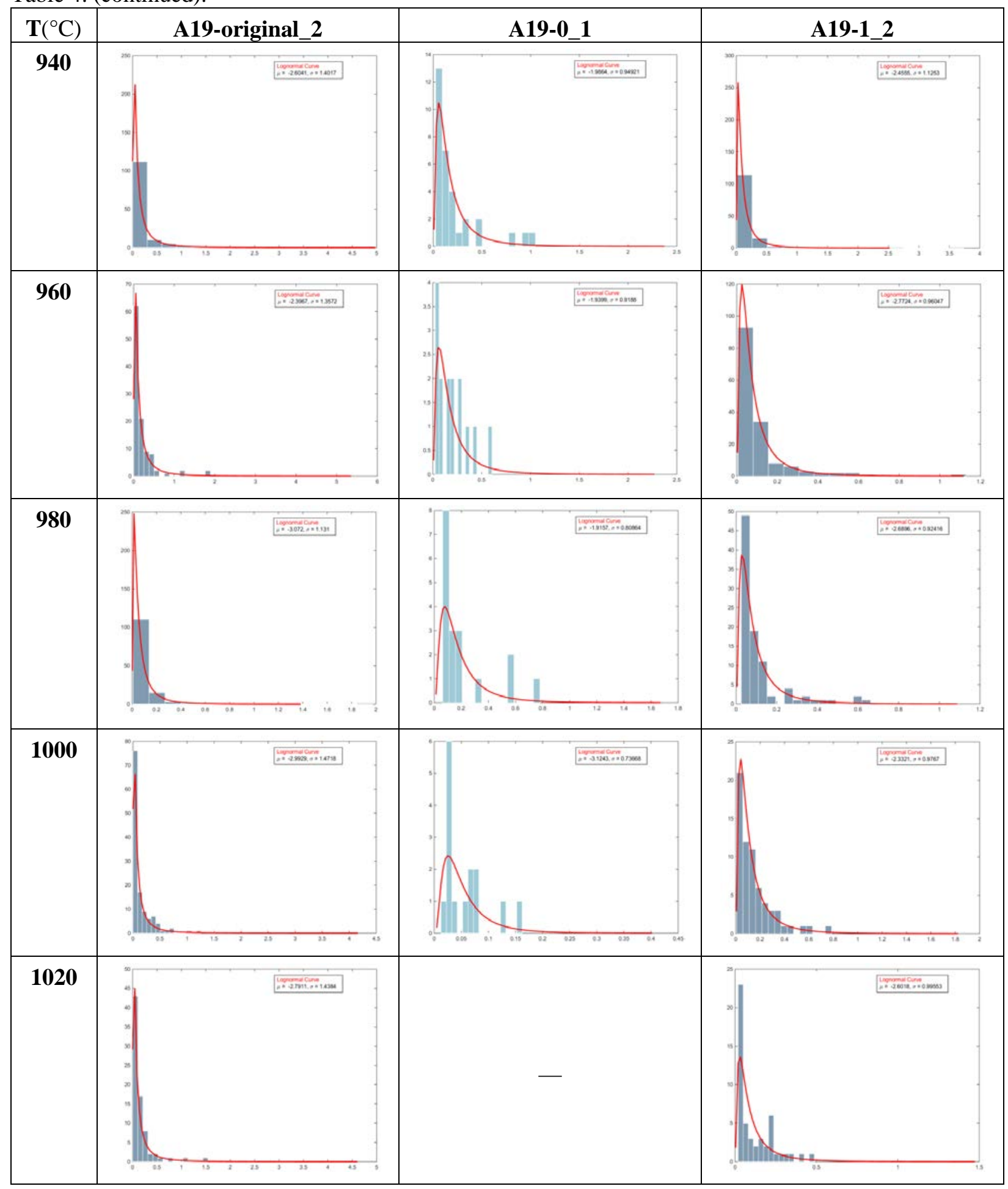


Table 4. (continued).

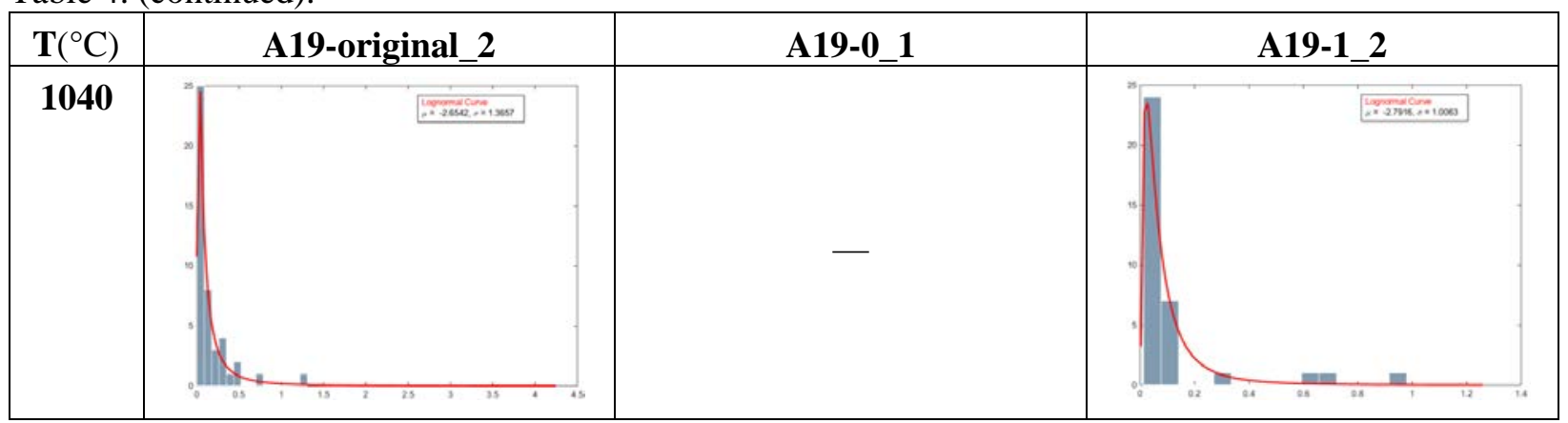

Figure 10 and Figure 11 provide boxplots of equivalent feature diameter and feature size as a function of temperature for each pellet composition. These help to identify outliers as well as compare distributions. It is shown in Figure 10 that overall the distributions show a right skewed trend. While similar to the other compositions, the composition A19-Original_2 has a greater number of outlying large bubbles. The figures show an increase in distribution width from temperatures $720-800^{\circ} \mathrm{C}$ for A19-1_2 and A19-0_1, as well as an increase in distribution width from temperatures $720-820^{\circ} \mathrm{C}$ for A19Original_2.
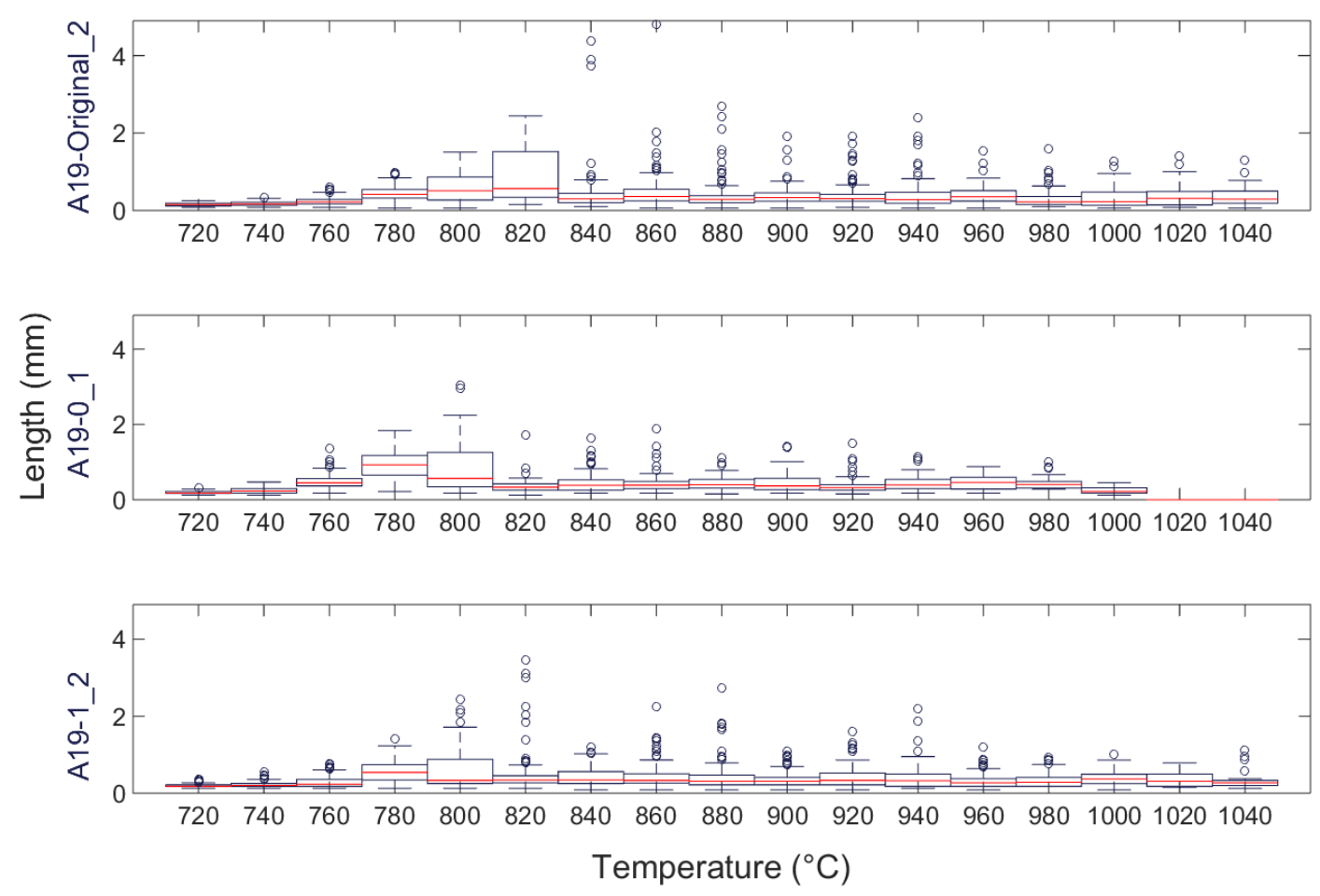

Figure 10. Equivalent Feature Diameter Boxplots. 

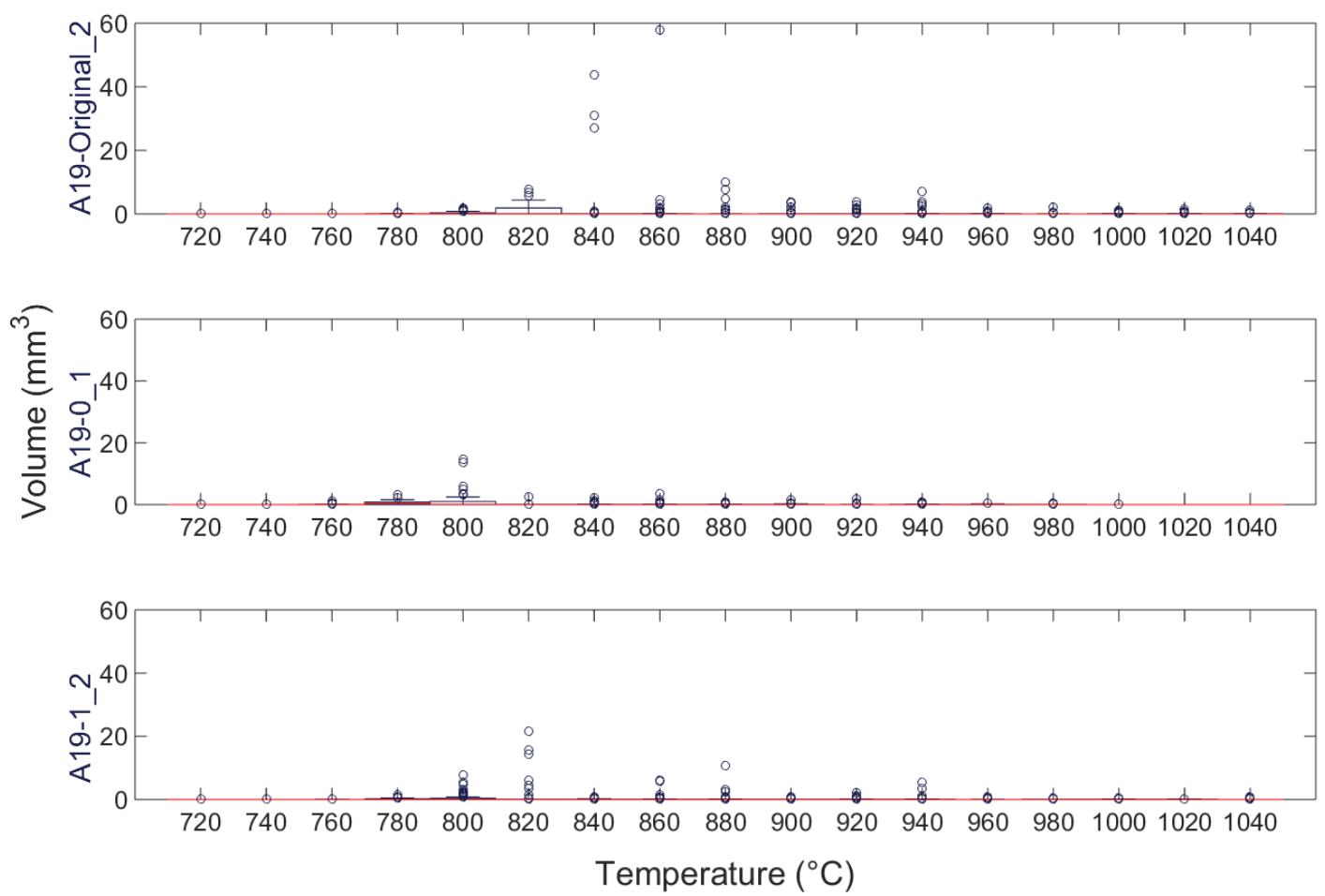

Figure 11. Equivalent Feature Sphere Volume Boxplots.

\subsection{Total Pellet Volume and Void Fraction}

Total Pellet Volume is computed with the assumption that the pellet shape is perfectly round, that the right side of the image is accurate and that the pellet is level. Using MATLAB code Appendix B, Subsection B-2, segmented images are imported, bubbles are removed, and the boundary of the pellet is traced using the built-in function bwboundaries(). Since only one side of the image is needed to compute the total volume of the pellet, the center of the pellet is found and only the points on the right side of the image are used. These boundary points are then approximated by a $8^{\text {th }}$ degree polynomial (with robust linear least-squares fitting), which is then rotated to compute the total volume of the pellet using what is commonly known as the Disk Method. A complication found with images of A19-Original_2 is that the ends of the pellet at temperatures $880-1040^{\circ} \mathrm{C}$ are cut off. This issue is resolved by simply extrapolating the curve using a first order polynomial fitted to the last 15 boundary data points before the cut off. Figure 12 shows the example of this curve fitting, with the polynomial fit in outlined in blue, and the linear fit in red. This procedure allows for a more accurate calculation of the total pellet volume for all of the images. Figure 13 plots the total pellet volumes obtained using this method against temperature for the three pellet compositions analyzed. From this plot we encounter some interesting discoveries, particularly in the sharp decrease in total pellet volume at $820^{\circ} \mathrm{C}$ and $840{ }^{\circ} \mathrm{C}$ for pellets A19-0_1 and A19-1_2 respectively. One speculation as to the cause of this abrupt decrease is attributed to the sudden release of gases. It is also seen that composition A19-0_1 has an overall lower total volume than A19-Original_2, and though A19-1_2 has the highest initial total volume, it is lower than that of A19-Original_2 over the ranges $600-660^{\circ} \mathrm{C}$ and $780-1040^{\circ} \mathrm{C}$, and at $1040^{\circ} \mathrm{C}$ has the lowest total volume. Overall, all three of the pellets show an increase in the volume from about $700^{\circ} \mathrm{C}$ up to about $840^{\circ} \mathrm{C}$, followed by a steady decline in the volume as temperature in increased. The increase in volume is due to the some of the gas evolved during the conversion process becoming trapped within the pellets above $700^{\circ} \mathrm{C}$. Above this temperature high viscous and surface tension forces of the feed cause the retention of the evolved gas. As the temperature is further increased beyond $840^{\circ} \mathrm{C}$, gases begin escaping the pellet and the volume begins to drop. The decrease in the viscosity as a function of temperature ${ }^{3}$ decreases the viscous forces and is likely 
the cause for allowing the bubbles to escape the pellet more readily. The model-estimated viscosities for these feeds show a decreasing trend from A19-Original to A19-1 to A19- $0^{5}$, which is consistent with this reasoning. The effect of swelling at low temperatures between $300^{\circ} \mathrm{C}$ and $600^{\circ} \mathrm{C}$ shown in the study by Lee et al. is not represented in the Figure 13, as those temperature images were not included in the x-ray tomography data provided.

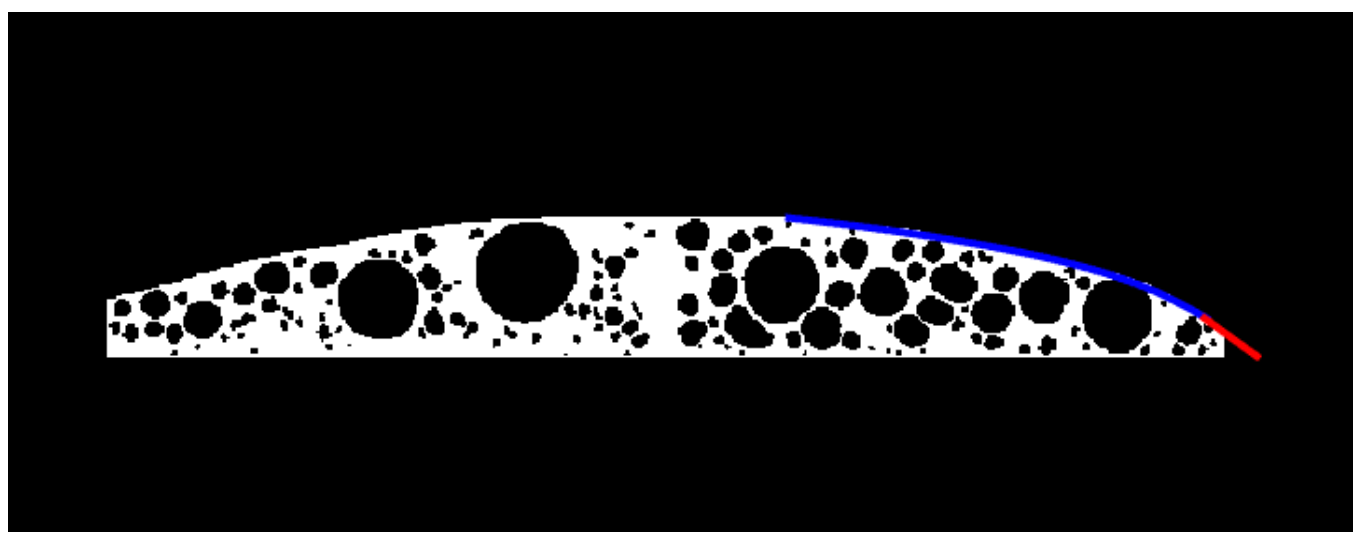

Figure 12. Total Pellet Volume Curve Fit and Extrapolation.

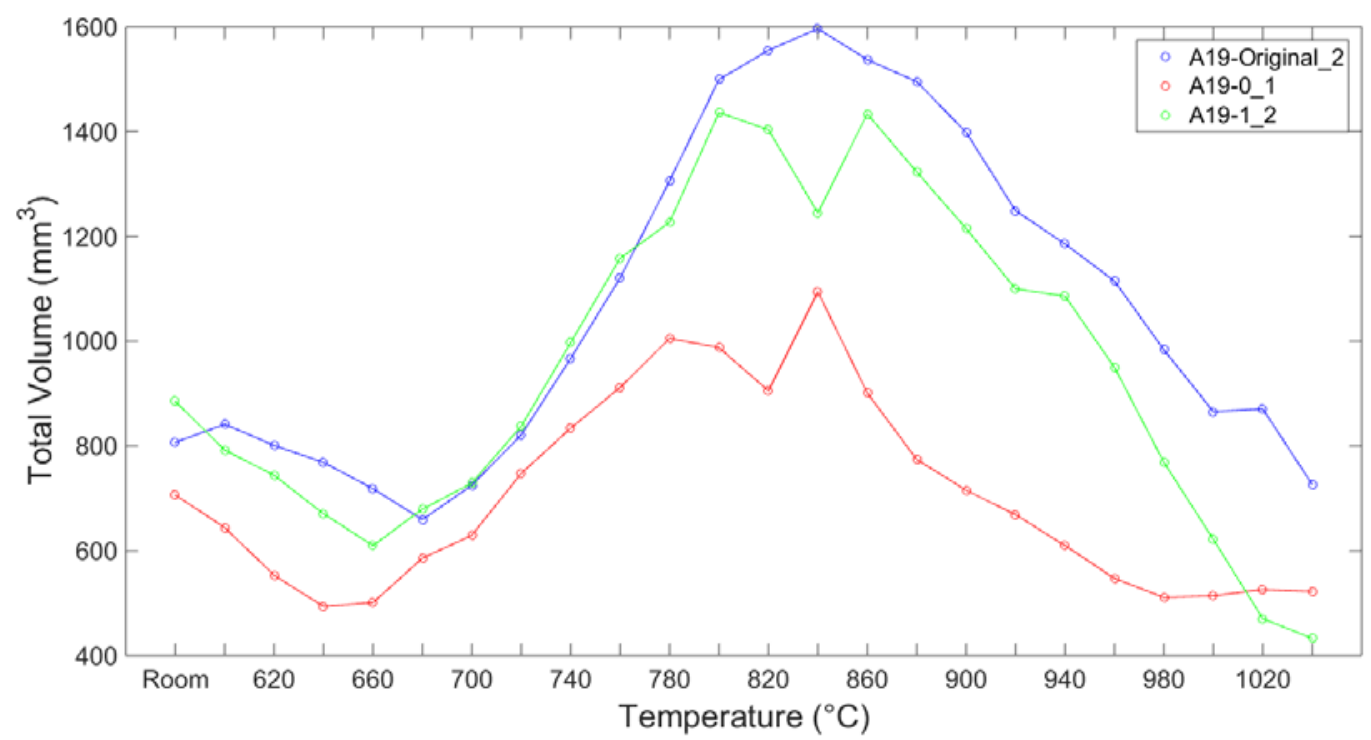

Figure 13. Total Pellet Volume vs. Temperature.

The advantage to the analysis of images created with x-ray tomography is that the void fraction, of the feed samples can also be examined. Total pellet volume is additionally used in the calculation of void fraction $(\phi)$, as defined by the ratio; $\phi=\frac{V_{V}}{V_{T}}$, where $V_{v}$ is the volume of void-space (or sum of bubble volume) and $V_{T}$ is the total volume. Currently, the value of $V_{v}$ is simply the sum of bubble volumes computed from the data shown in the images and corresponding lognormal distributions. Figure 14 shows the calculated void fraction of the three pellets against temperature. An essential note about this calculation is, as the data accounts for only the center segment of the pellet the actual porosity would be much higher than depicted, this would be completed using the volume distributions discussed in Section A, however the same trend would be expected. For a direct comparison with the method of Hilliard and Hrma $^{6}$, this step of the analysis needs to be completed. The pellets initial void fraction until 
approximately $720^{\circ} \mathrm{C}$ remains at 0 due to no recordable bubbles present in this temperature range, then increases, peaking at 840,800 and $820^{\circ} \mathrm{C}$ with void fraction values of $0.0671,0.0519$, and 0.0516 for A19-Original_2, A19-0_1, and A19-1_2 respectively. Void fraction then decreases sharply and oscillates, descending to values 0.0048 for A19-Original_2 and A19-1_2 and 0 for A19-0_1 at 1040. Similar to what was shown in Figure 13, the void fraction of A19-Original_2 is higher than A19-0_1 and A19-1_2 over the majority of the temperature range, this is due to what was previously shown in Figure 8 and Figure 9 with higher bubble diameter and volume. Additionally, there is an apparent shift in the graph from A19-0_1 to A19-1_2 by 20 degrees, maintaining a similar shape. This shift in the peak void fractions of the three pellets is likely a direct cause of the viscosities of the feeds. The lowest temperature peak occurs with A19-0_1, which has the lowest model-estimated viscosity, and the highest temperature peak occurs with the A19-original_2, which has the highest viscosity. The void fraction of the feed pellets will have a direct correlation with two of the important physical parameters of the cold cap model, the effective density and the thermal conductivity.

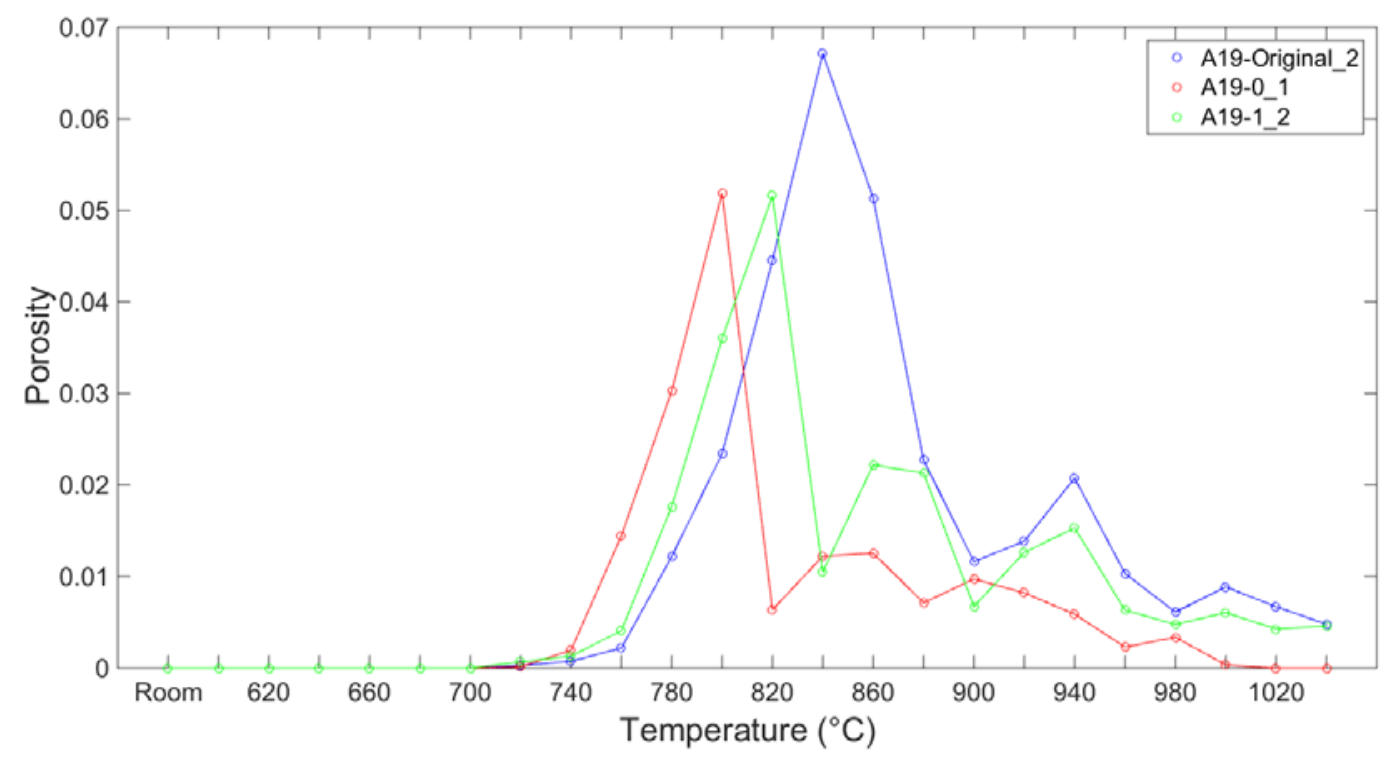

Figure 14. Void Fraction vs. Temperature based on 2D estimations.

\section{FUTURE WORK}

In conclusion, though we have been able to perform preliminary analysis of pellet compositions A19-0_1, A19-1_2, and A19-original_2, it is essential we develop a more efficient method of image segmentation to complete the remaining three compositions as well as for future experiments. INL plans to complete the image segmentation and analysis for the remaining 3 compositions as well as a comprehensive analysis of all six compositions. Plans for this additional analysis will include comparison with the measurements performed at PNNL to see how the approach of measuring 2D profiles matches with 3D X-ray data. The information will then further be used to synthetically generate a cold cap structure using DREAM.3D that can be used to model heat transfer through the cold cap layer of waste glass melter for a particular melter feed. Future documentation will also contain further information about the experimental measurements used in the 3D X-ray images. 


\section{REFERENCES}

$1 \quad$ R. A. Kirkbride, et al., Tank Waste Remediation System Operation and Utilization Plan, Vol. I, HNF-SD-WM-SP-012, Numatec Hanford Corporation, (Richland, Washington, 1999).

2 Z. Hilliard and P. Hrma, “A Method for Determining Bulk Density, Material Density and Porosity of Melter Feed During Nuclear Waste Vitrification,” J. Am. Ceram. Soc., 99, 98-105 (2016).

3 R. Pokorny, Z.J. Hilliard, D.R. Dixon, M.J. Schweiger, D.P. Guillen, A.A. Kruger and P. Hrma. “One dimensional cold cap model for melters with bubblers,” Journal of American Ceramic Society, 98(10), pp. 3112-3118, (2015).

4 F. C. Johnson, A. S. Choi, D. H. Miller, and D. M. Immel, Comparison of HLW Glass Melting Rate Between Frit and Glass Forming Chemicals Using X-Ray Computed Tomography, SRNL-STI-2014-00562 Revision 0, Aiken, SC, April 2015, U. S. Department of Energy (2015).

5 J. Marcial, J. Chun, P. Hrma and M. Schweiger, Effect of Bubbles and Silica Dissolution on Melter Feed Rheology during Conversion to Glass, Environ. Sci. Tech., Vol 48, pp. 12173-12180, 2016.

$6 \quad$ K.S. Matlack, H. Gan, M. Chaudhuri, W. Kot, W. Gong, T. Bardakci, and I.L. Pegg. DM100 and DM1200 Melter Testing with High Waste Loading Glass Formulations for Hanford High-Aluminum HLW Streams, VSL-10R1690-1, Rev 0, Vitreous State Laboratory, The Catholic University of America, 8/16/2010.

7 S. Lee, Z. Hilliard, J.S. Heilman-Moor, C.C. Bonham, D.R. Dixon, P. Hrma, M.J. Schweiger, A.A. Kruger, The Effect of Quartz Size and Melt Viscosity on HLW Melter Feed Using Pellet Test Response, WM2016 Conference, March 6-10, 2016, Phoenix, Arizona.

$8 \quad$ K. Watanabe, T. Yano, K. Takeshita, K. Minami and E. Ochi, X-ray CT Imaging of Vitrified Glasses Containing Simulant Radioactive Wastes: Structure and Chemical Reactions of Glass Beads and Wastes in the Cold Cap, Glass Technol.: Eur. J. Glass Sci. Technol. A, Vol 53, pp. 273-278, (2012).

$9 \quad$ S. Jean. Introduction to mathematical morphology. Computer vision, graphics, and image processing 35.3 (1986): 283-305.

10 S. Jean, and P. Soille, eds., Mathematical morphology and its applications to image processing. Vol. 2. ,Springer Science \& Business Media, 2012.

11 M.V. Glazoff, S.N. Rashkeev, Y.P. Pyt'ev, J.W. Yoon, and S. Sheu, Interplay between plastic deformations and optical properties of metal surfaces: A multiscale study, Applied Physics Letters, 95(8), 084106, (2009).

12 M. Groeber and M. Jackson, DREAM.3D: A Digital Representation Environment for the Analysis of Microstructure in 3D, Integrating Materials and Manufacturing Innovation, 3:5, December 2014. 


\section{Appendix A}

\section{Dream.3D Data Extraction Pipeline}

[1] ITK: Import Images (3D Stack)

[2] Extract Component as Attribute Array

[3] Threshold Objects

[4] Create Data Array

[5] Replace Value in Array (Conditional)

[6] Export Data (ASCII Text)

[7] Segment Features (Scalar)

[8] Find Feature Phases

[9] Create Attribute Matrix

[10] Find Feature Neighbors

[11] Find Feature Centroids

[12] Find Surface Features

[13] Create Data Array

[14] Find Feature Sizes

[15] Find Feature Shapes

[16] Find Feature Neighborhoods

[17] Generate Ensemble Statistics

[18] Find Volume Fractions of Ensembles

[19] Export Data (ASCII Text)

[20] Write DREAM.3D Data File

[21] Write Feature Data as CSV File 


\section{Appendix B}

\section{MATLAB Scripts \\ B-1. MATLAB READ DREAM.3D OUTPUT}

\%\% IMPORT DREAM.3D DATA TO MATLAB and MAKE HISTOGRAMS

\% This code is for the purpose of reading Dream3D .CSV data, that has been condensed into

$\%$ a single Excel sheet, into Matlab. This code will also generate hisograms of Bubble Size

$\%$ and Equivalent Diamgeter for each temperature. The Excel file needed can be found on the

\% google drive 'Melter> YanoCT > February2016 Images' in the 'Image Data Excel Files' folder for

$\%$ the particular pellet composition.

\%\% Parameters to Change

workbookFile = 'A19-original_2_All Temperatures.xlsx';

sheetName = 'A19-original_2_'; \%begining of sheet name excluding number

titleName = 'A19-Original \2'; \%must include ' ' before any '_,

pathname $=$ 'C: $\backslash$ Users $\backslash$ MITCLE $\backslash$ Documents $\backslash A 19-O r i g i n a l \_2 〉 ’$ \%save path

VoxelSize $=0.052 ; \% \mathrm{~mm} / \mathrm{voxel}$

totalsheets $=24$; \%Identify total number of sheets to read through

startingTemp $=600$;

\%plotColor $=$ [158 202 215] ./ 255; \%Blue

plotColor $=\left[\begin{array}{ll}129 & 155 \\ 177\end{array}\right]$./ 255; \%Grey

$\% \%$ Allocate cell Array to store full data sets

Data = cell(1,totalsheets);

$\% \%$ Begin loop through sheets

for num $=1$ :totalsheets

$\% \%$ Input handling

sheetNamefull= char(strcat(sheetName,num2str(num),'.csv'));

Bubblescount = xlsread(workbookFile, sheetNamefull, 'A1:A1');

startRow = 3;

endRow $=$ Bubblescount +2 ;

\%\%Generate Graph Title Temperature

if num $==1$

tabtitle $=\operatorname{char}\left(\operatorname{strcat}\left(\right.\right.$ titleName, $\left\{\right.$ ' $\left.\left.\left.^{\text {Room' }}\right\},\left\{{ }^{\circ} \mathrm{C}^{\prime}\right\}\right)\right)$;

else

tabtitle = char(strcat(titleName, $\left\{{ }^{\prime}\right.$ ' $\}$, num2str(startingTemp), $\left.\left\{{ }^{\circ}{ }^{\circ} \mathrm{C}\right\}\right)$ );

end

if Bubblescount $>2 \%$ Only continue if there are bubbles

$\% \%$ Import the data

$[\sim, \sim$, raw $]=$ xlsread(workbookFile, sheetNamefull, sprintf('A\%d:W\%d',startRow(1),endRow(1)));

for block=2:length(startRow)

$[\sim, \sim$, tmpRawBlock $]=$ xlsread(workbookFile, sheetNamefull,

sprintf('A\%d:W\%d',startRow(block), endRow(block)));

raw $=[$ raw;tmpRawBlock $] ; \% \#$ ok $<$ AGROW $>$

end

raw(cellfun(@(x) isempty(x) \&\& isnumeric(x) \&\& isnan(x),raw)) = \{“‘ $\}$; 
\%\% Replace non-numeric cells with $\mathrm{NaN}$

$\mathrm{R}=$ cellfun(@(x) isnumeric(x) \&\& islogical(x),raw); \% Find non-numeric cells

$\operatorname{raw}(\mathrm{R})=\{\mathrm{NaN}\} ; \%$ Replace non-numeric cells

$\% \%$ Create output variable

$\mathrm{I}=$ cellfun(@(x) ischar(x), raw);

$\operatorname{raw}(\mathrm{I})=\{\mathrm{NaN}\}$;

data $=$ reshape $([\operatorname{raw}\{:\}], \operatorname{size}($ raw $))$;

$\% \%$ Allocate imported array to column variable names, save to cell array

$[\sim, \mathrm{I}]=\max (\operatorname{data}(:, 22)) ; \%$ Find the largest attribute (non bubble area)

data(I,:)=[]; \%Remove the row from data set

$[\sim, I]=\max (\operatorname{data}(:, 22)) ; \%$ Find the largest attribute (non bubble area)

data(I,:)=[]; \%Remove the row from data set

data(:,15) = data(:,15)*VoxelSize;

EquivalentDiameters = data(:,15);

data(:,22) = data(:,22)/((1/VoxelSize $) *(1 /$ VoxelSize $)$;

SizeVolumes = data(:,22);

data(:,24) $=(4 / 3) * \mathrm{pi}^{*}(($ EquivalentDiameters/2).^3);

Data $\{$ num $\}=$ data; \% Save into cell array

$\% \%$ Create Histogram of Attribute Area get Attribute Summary

figure(1)

[p,d] = histfitmod(SizeVolumes,15,'lognormal');

histtitle $=\operatorname{char}\left(\operatorname{strcat}\left(\left\{{ }^{\prime}\right.\right.\right.$ Bubble Area Histogram ' $\}$,tabtitle, $\left\{\right.$ ' $\left(\mathrm{mm}^{\wedge} 2\right)$ ' $\left.\left.\}\right)\right)$;

title(histtitle)

ylabel('Frequency')

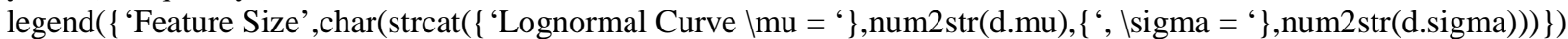

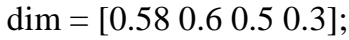

str $=\left\{{ }^{\prime}\{\mid\right.$ color $\{$ red $\}$ Lognormal Curve $\}$ ', $\operatorname{char}\left(\operatorname{strcat}\left(\left\{{ }^{\prime} \mid \operatorname{mu}={ }^{\prime}\right\}\right.\right.$, num2str(d.mu), $\left\{{ }^{\prime}, \backslash\right.$ sigma $\left.={ }^{\prime}\right\}$, num2str(d.sigma $\left.\left.\left.)\right)\right)\right\}$;

annotation('textbox',dim,'String',str,'FitBoxToText','on');

h = findobj(gca,'Type','patch');

h.FaceColor = plotColor;

h.EdgeColor = ' $w$ ';

\%\%Save Histogram to Specified Folder

saveas(figure(1),fullfile(pathname,char(strcat(strrep(histtitle,'’,'’),'.png'))))

clf

$\% \%$ Create Histogram of Equivilant Diameter

figure(2)

[p,d] = histfitmod(EquivalentDiameters,15,'lognormal');

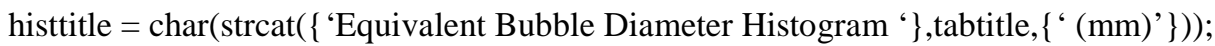

title(histtitle)

ylabel('Frequency')

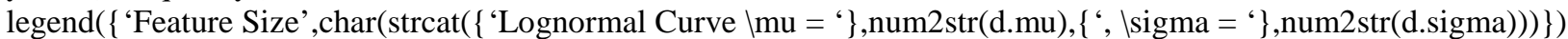

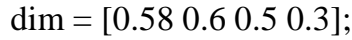

str $=\left\{{ }^{\prime}\{\backslash\right.$ color $\{$ red $\}$ Lognormal Curve $\}$ ', $\operatorname{char}\left(\operatorname{strcat}\left(\left\{{ }^{\prime} \mid \operatorname{mu}={ }^{\prime}\right\}\right.\right.$, num2str(d.mu), $\left\{{ }^{\prime}, \backslash \operatorname{sigma}={ }^{\prime}\right\}$, num2str(d.sigma $\left.\left.\left.)\right)\right)\right\}$;

annotation('textbox',dim,'String',str,'FitBoxToText','on');

h = findobj(gca,'Type','patch');

h.FaceColor = plotColor;

h.EdgeColor = ' $w$ '; 
\%\%Save Histogram to Specified Folder

saveas(figure(2),fullfile(pathname,char(strcat(strrep(histtitle,'’,,'),'.png'))))

clf

$\% \%$ Save and clear Workspace

end

if num $>1$

startingTemp $=$ startingTemp +20 ;

end

end

\section{B-2. MATLAB TOTAL VOLUME GENERATION}

$\% \%$ TOTAL PELLET VOLUME

$\%$ This code is for the purpose of generating total pellet volume, to be used in

$\%$ the computation of porosity. The directory must contain the folder of

\% segmented images titled in resemblance to 'XZ planes of A19-0_1_1full.png'

$\%$ these can be found on the google drive 'Melter> YanoCT > February2016

\% Images' in the 'Clean Images > Full' folder for the particular pellet

$\%$ composition.

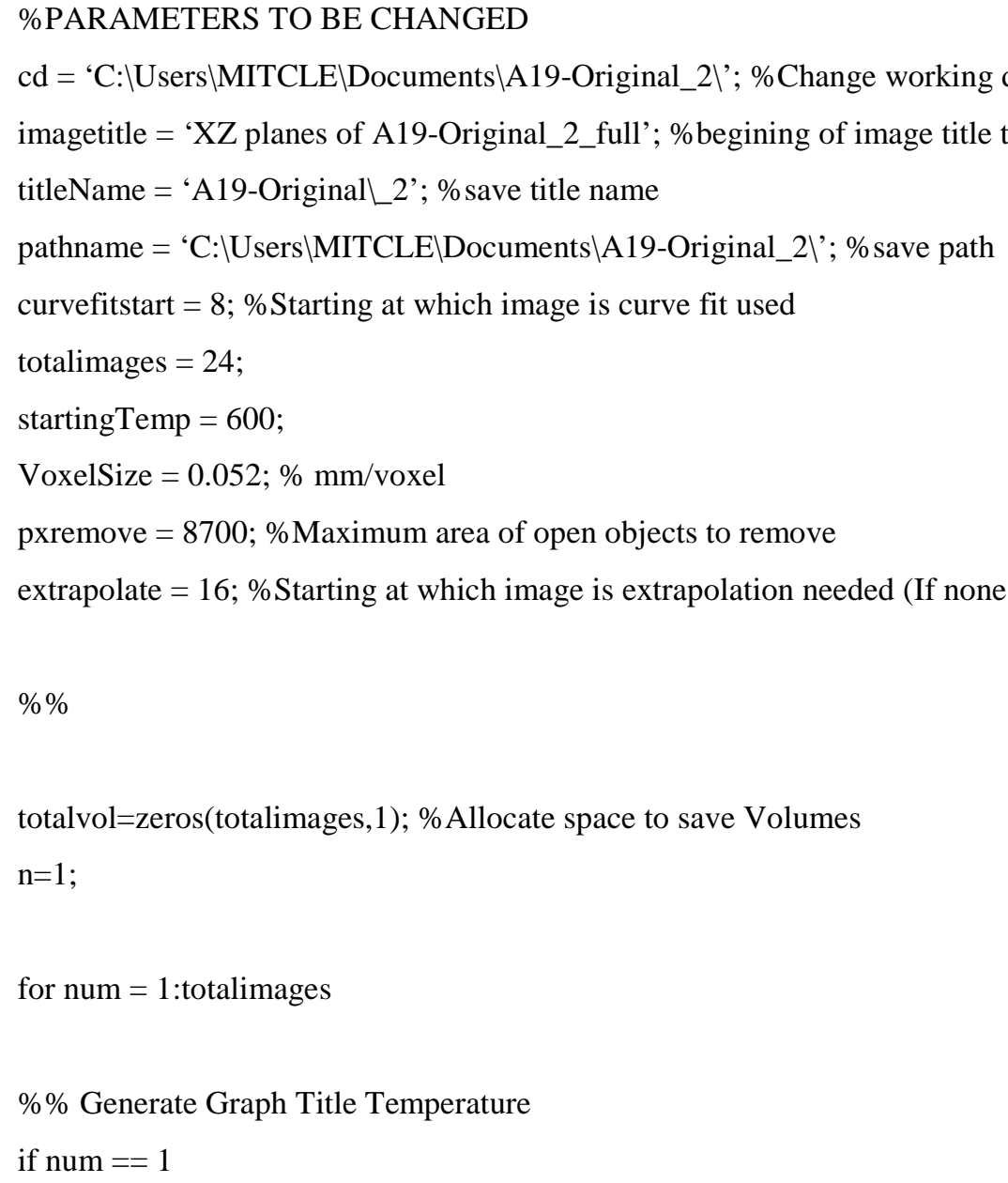


graphtitle $=\operatorname{char}\left(\right.$ strcat(titleName, $\left\{{ }^{‘}\right.$ Room’ $\left.\left.\},\left\{{ }^{\circ}{ }^{\circ} ’\right\}\right)\right)$;

else

graphtitle $=\operatorname{char}\left(\operatorname{strcat}\left(\right.\right.$ titleName, $\{$ ‘ ' $\}$, num2str(startingTemp), $\left.\left.\left\{{ }^{\circ}{ }^{\circ} \mathrm{C}\right\}\right)\right)$;

end

\%\% Read image, Remove Bubbles, Find Boundary points

RGB = imread(char(strcat(imagetitle,num2str(num),'.png'))); \%Read Image

threshold = graythresh(RGB); \%Threshold Image

bw = im2bw(RGB,threshold); \%Convert to binary

bw = bwareaopen(bw,pxremove); \%Remove all open objects containing fewer than a set number of pixels

bw = imcomplement(bw); \%Take the compliment of the image

bw = bwareaopen(bw,pxremove); \%Remove all open objects containing fewer than a set number of pixels

bw = imcomplement(bw); \%Take the compliment of the image

figure(1)

imshow(bw) \%Show Image

saveas(figure(1),fullfile(pathname,char(strcat(imagetitle,num2str(num),'fullnobubbles.png'))))

clf

bw = flipdim(bw ,1);

[B,L] = bwboundaries(bw,'noholes'); \%Get boundary points

$\%$ hold on \%used to plot boundary

for $\mathrm{k}=1$ :length(B)

boundary $=\mathrm{B}\{\mathrm{k}\}$;

\%plot(boundary(:,2),boundary(:,1),'r','Linewidth',2) \%used to plot boundary

end

\% Note: boundary(:,1) = Y-axis and boundary(:,2) $=$ X-axis

ycordmin = min(boundary(:,1)); \%find bottom edge

ycordmax $=\max ($ boundary(:,1)); \%find the top edge

xcordmin $=\min ($ boundary(:,2)); \%find left edge

xcordmax = max (boundary(:,2)); \%find right edge

$\mathrm{xmin}=((\mathrm{xcordmax}-\mathrm{xcordmin}) / 2)+\mathrm{xcordmin} ; \%$ find center of image

rows_to_remove = any (boundary==ycordmin, 2); \%determine points to remove bottom edge

boundary(rows_to_remove,:) = []; \%Remove bottom edge

boundary(boundary(:,2)<xmin,:) = [];\%Remove $\mathrm{x}$ values lower than center of image

\%set image origin to 0,0 and convert to $\mathrm{mm}$

for $\mathrm{i}=1$ :length(boundary)

boundary $(\mathrm{i}, 2)=($ boundary $(\mathrm{i}, 2)-\mathrm{xmin}) *$ VoxelSize;

boundary $(\mathrm{i}, 1)=($ boundary $(\mathrm{i}, 1)-\mathrm{ycordmin}) *$ VoxelSize;

end 
$[\sim, \mathrm{I}]=\operatorname{sort}($ boundary $(:, 2))$; boundarynew=boundary $(\mathrm{I},:) ; \%$ sort and reorder boundary points $\mathrm{x}$-axis boundarynew(:,1)= flipud(boundarynew(:,1)); \%reverse boundary points y-axis

\%\% Curve Fitting, Extrapolation, Total Volume Calculation

if num $<$ curvefitstart

radius $=(($ xcordmax $-($ xcordmin-1) $) / 2) *$ VoxelSize;

height $=(\text { ycordmax }-(\text { ycordmin }-1))^{*}$ VoxelSize;

totalvol $(\mathrm{n})=\mathrm{pi}^{*}\left(\right.$ radius $\left.^{\wedge} 2\right) *$ height; $\%$ Formula for volume of a clyinder

elseif num >= curvefitstart $\& \&$ num $<$ extrapolate

f=fit(boundary(:,1),boundary(:,2),'poly8','Robust','Bisquare’); \%fit curve to boundary points

figure (2)

plot(f,boundary(:,1),boundary(:,2),'*')

xlim([0,16]); ylim([0,16]); view(-90,90); set(gca,'ydir','reverse')

plottitle $=\operatorname{strrp}(\operatorname{char}(\operatorname{strcat}(\{$ 'Boundary Curve Fit' $\}$, graphtitle, \{'Voxels' $\})),{ }^{\prime} \backslash$, ,' $)$;

figfile = fullfile(pathname,char(strcat(plottitle,'.png')));

saveas(figure(2),figfile)

clf

\%Compute Volume

totalvol(n)=integral(@(x) pi*(f(x).^2),0,max(boundary(:,1)),'ArrayValued’, true);

else

boundarynew = boundary;

indices $=$ find $($ boundarynew $(:, 2)==\max ($ boundarynew $(:, 2)))$;

boundarynew(indices,:)=[];

f1=fit(boundarynew(:,1),boundarynew(:,2),'poly5','Robust','Bisquare'); \%poly5 is default boundarynnew = boundarynew(1:15,:); \%Use the last 15 points to fit extrapolation

f2=fit(boundarynnew(:,1),boundarynnew(:,2),'poly1','Robust', 'Bisquare'); \%poly1 is default figure (2)

plot(boundary(:,1),boundary(:,2),'*’)

hold on

fplot(f1,[min(boundarynnew(:,1)),16],'r')

fplot(f2,[0,min(boundarynnew(:,1))],'b’)

xlim([0,16]); ylim([0,16]); view(-90,90); set(gca,'ydir','reverse')

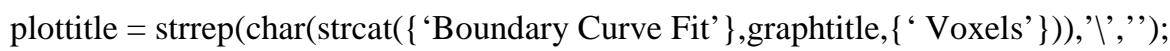

figfile = fullfile(pathname, char(strcat(plottitle,'.png')));

saveas(figure(2),figfile)

clf

\%Compute Volume 
totalvol(n)=integral(@(x) pi*(f1(x).^2),min(boundarynnew(:,1)),max(boundary(:,1)),'ArrayValued', true)+... integral(@(x) pi*(f2(x).^2),0,min(boundarynnew(:,1)),’ArrayValued', true);

end

if num $>1$

startingTemp $=$ startingTemp +20 ;

end

$\mathrm{n}=\mathrm{n}+1$;

end 